\title{
Epidemiological Predictive Modeling of COVID-19 Infection: Development, Testing, and Implementation on the Population of the Benelux Union
}

\section{OPEN ACCESS}

Edited by:

Yousef Saleh Khader,

Jordan University of Science and

Technology, Jordan

Reviewed by:

Susanta Kumar Ghosh,

National Institute of Malaria Research

(ICMR), India

Davide Baroli,

RWTH Aachen University, Germany

Vishal Deo,

University of Delhi, India

Julio Elias Normey-Rico,

Federal University of Santa

Catarina, Brazil

*Correspondence:

Nenad Filipovic

fica@kg.ac.rs

Specialty section:

This article was submitted to Infectious Diseases - Surveillance,

Prevention and Treatment,

a section of the journal

Frontiers in Public Health

Received: 22 June 2021

Accepted: 23 September 2021

Published: 28 October 2021

Citation:

Šušteršič T, Blagojević A, Cvetković $D$, Cvetković A, Lorencin I, Šegota SB, Milovanović D, Baskić D, Car Z and

Filipović N (2021) Epidemiological

Predictive Modeling of COVID-19 Infection: Development, Testing, and Implementation on the Population of the Benelux Union.

Front. Public Health 9:727274. doi: 10.3389/fpubh.2021.727274

\begin{abstract}
Tijana Šušteršič ${ }^{1,2}$, Andjela Blagojević ${ }^{1,2}$, Danijela Cvetković ${ }^{3}$, Aleksandar Cvetković ${ }^{4}$ Ivan Lorencin ${ }^{5}$, Sandi Baressi Šegota ${ }^{5}$, Dragan Milovanović ${ }^{6,7}$, Dejan Baskić ${ }^{7,8}$, Zlatan Car $^{5}$ and Nenad Filipović ${ }^{1,2 *}$

${ }^{1}$ Faculty of Engineering, University of Kragujevac, Kragujevac, Serbia, ${ }^{2}$ Bioengineering Research and Development Center (Bio/RC), Kragujevac, Serbia, ${ }^{3}$ Institute for Information Technologies, University of Kragujevac, Kragujevac, Serbia, ${ }^{4}$ Department of Surgery, Faculty of Medical Sciences, University of Kragujevac, Kragujevac, Serbia, ${ }^{5}$ Faculty of Engineering, University of Rijeka, Rijeka, Croatia, ${ }^{6}$ Clinical Centre Kragujevac, Kragujevac, Serbia, ${ }^{7}$ Faculty of Medical Sciences, University of Kragujevac, Kragujevac, Serbia, ${ }^{8}$ Institute of Public Health Kragujevac, Kragujevac, Serbia
\end{abstract}

Since the outbreak of coronavirus disease-2019 (COVID-19), the whole world has taken interest in the mechanisms of its spread and development. Mathematical models have been valuable instruments for the study of the spread and control of infectious diseases. For that purpose, we propose a two-way approach in modeling COVID-19 spread: a susceptible, exposed, infected, recovered, deceased (SEIRD) model based on differential equations and a long short-term memory (LSTM) deep learning model. The SEIRD model is a compartmental epidemiological model with included components: susceptible, exposed, infected, recovered, deceased. In the case of the SEIRD model, official statistical data available online for countries of Belgium, Netherlands, and Luxembourg (Benelux) in the period of March 152020 to March 152021 were used. Based on them, we have calculated key parameters and forward them to the epidemiological model, which will predict the number of infected, deceased, and recovered people. Results show that the SEIRD model is able to accurately predict several peaks for all the three countries of interest, with very small root mean square error (RMSE), except for the mild cases (maximum RMSE was $240.79 \pm 90.556$ ), which can be explained by the fact that no official data were available for mild cases, but this number was derived from other statistics. On the other hand, LSTM represents a special kind of recurrent neural network structure that can comparatively learn long-term temporal dependencies. Results show that LSTM is capable of predicting several peaks based on the position of previous peaks with low values of RMSE. Higher values of RMSE are observed in the number of infected cases in Belgium (RMSE was 535.93) and Netherlands (RMSE was 434.28), and are expected because of thousands of people getting infected per day in those countries. In future studies, we will extend the models to include mobility information, variants of concern, as well as a medical intervention, etc. A prognostic model could help us predict epidemic peaks. In that way, we could react in a timely manner by introducing new or tightening existing measures before the health system is overloaded.

Keywords: COVID-19, disease spread modeling, SEIRD model, LSTM model, epidemiological model 


\section{INTRODUCTION}

Wuhan was registered on December 19, 2019 as the epicenter of the emergence of a new virus from the group of coronaviruses that showed the characteristics of inter-human transmission, causing a respiratory disease presenting with fever, dry cough, and, often, severe pneumonia with acute respiratory distress syndrome (ARDS) (1). The World Health Organization (WHO) announced the pandemic disease Coronavirus Disease-2019 (2) caused by severe acute respiratory syndrome coronavirus 2 (SARS-CoV-2) and brought measures in order to interrupt the spread of SARS-CoV-2 worldwide. Emerging and recurring diseases have contributed to a renewed interest in infectious diseases. Mathematical models have been valuable instruments for the study of the spread and control of infectious diseases. Understanding the dynamics of transmission of infectious diseases in populations, regions, and countries will contribute to better approaches to reducing the spread of these diseases (3-6). Indeed, the number of infected people grows exponentially, and many countries have decided to impose a complete lockdown of affected cities in order to reduce the number of contacts and stop the spread of the virus (7).

To this end, several approaches have been suggested to predict the spread of COVID-19 in population. Compared with standard statistical methods $(8,9)$, mathematical models based on dynamic equations (10-12) attract comparatively less consideration, although they may provide more insight into the dynamics of epidemics (11). Modeling of infectious diseases is most conveniently performed using deterministic compartmental models. Adjusting the parameters of the equations allows better modeling of environmental characteristics, such as social restrictions (7). These models are based on flow patterns between compartments such as susceptible (S), exposed (E), infected (I), and recovered $(\mathrm{R})$; therefore, their names are often referred to as SEIR, SIT, SIRS, etc. Among all these models, the classical susceptible, expose, infectious, recovered (SEIR) model is the most commonly used concept for characterizing the epidemic of COVID-19 in both China and other countries (11). On the basis of the SEIR model, the success of different interventions after the epidemic can be assessed (13-16), which seems to be a daunting challenge for general statistical techniques. One widely used model is the SIR (susceptible, infected, recovered) model for human-to-human transmission, which defines the migration of persons through three mutually incompatible periods of infection: prone, contaminated, and recovered (6). However, Yi-Cheng et al. assert that the traditional SIR model neglects time-varying properties, such as transmission and recovery rate (17). They discuss that the time-independent SIR model is too simple to precisely and effectively predict the trend of the disease. Therefore, they suggested a time-dependent SIR model, where both rates are functions of time $t$. Also, several models have been developed for the COVID-19 pandemic. Lin et al. have developed the SEIR (susceptible, released, infectious, deceased) model taking into account some parameter estimates from the 1918 influenza pandemic (18). Anastassopoulou et al. suggested a discrete SIR model with deceased individuals (19), Casella developed a control-oriented SIR model that highlights the consequences of delays and measures the results of various containment policies (20), and $\mathrm{Wu}$ et al. used propagation dynamics to measure the clinical magnitude of COVID-19 (21). Stochastic transmission models were also considered (22, 23). Giordano et al. suggest a new epidemiological mean-field approach for the COVID-19 outbreak in Italy, expanding the classic SIR model, close to that developed by Gumel et al. for SARS (24). The mentioned expanded SIR model, named SIDARTHE, considers eight stages of infection: susceptible, infected, diagnosed, ailing, recognized, threatened, healed, and deceased (6). The SIDARTHE model recognizes a difference among infected individuals depending on whether they have been diagnosed, and on the severity of their symptoms. The difference between diagnosed and non-diagnosed individuals is important, because the ones who have been diagnosed as positive are isolated, and it is less probable they will spread the infection. This delineation also helps to explain the incorrect interpretation of the epidemic spread and the case fatality rate (6).

In addition to the aforementioned models and variations, there are attempts in the literature to build agent-based models (ABMs) with various purposes and goals. Indeed, ABMs have long been used to simulate various illnesses $(25,26)$. As a result, ABMs have grown in popularity for modeling the spread of COVID-19 and analyzing alternative approaches to the problem (27-29). Notably, several studies have investigated the effects of contact tracing on the transmission of COVID-19 (30-33). Two most distinct and complete software in this are COVIDABS and Covasim. Silva et al. (34) developed a methodology for COVID-ABS, a novel SEIR (susceptible-exposed-infectedrecovered) $A B M$ that seeks to mimic pandemic dynamics by simulating individuals, businesses, and governments using a society of agents (34). Seven different scenarios of social distancing interventions were investigated, each with a different epidemiological and economic impact: (1) do nothing, (2) lockdown, (3) conditional lockdown, (4) vertical isolation, (5) partial isolation, (6) use of face masks, and (7) use of face masks in conjunction with $50 \%$ adhesion to social isolation. Kerr et al. (32) developed a methodology of Covasim (COVID-19 agent-based simulator) (35). Covasim, among others, supports a broad range of interventions, namely, non-pharmaceutical interventions like physical separation and protective equipment, pharmaceutical interventions like vaccination, and testing interventions like symptomatic and asymptomatic testing, isolation, contact tracing, and quarantine. Delays, loss-to-follow-up, microtargeting, and other variables can all be incorporated into these treatments. A use case was presented for Seattle/King County, Washington, United States from January 27 to November 14, 2020, with projections until December 31, including additional restrictions imposed on November 16. Its complexity proved to be adequate to examine epidemic dynamics and inform policy decisions.

Since epidemiological measures do not always give the expected and desired results and a pandemic is constantly changing its course, it is obvious that a new approach is necessary to improve the existing measures to fight the epidemic. Deep learning methods, such as recurrent neural networks (RNNs), 
are well-suited for modeling temporal sequences (36). However, the main limitation of RNNs is reflected in learning of longterm dependencies in large sequences that can involve hundreds or thousands of steps. These limitations are addressed by long short-term memory (LSTM) networks (37). There are also several studies that investigate LSTM as an approach to forecasting future COVID-19 cases (38-42). LSTM was used for COVID19 forecasting in Canada and achieved an accuracy of $93.4 \%$ for short-term and $92.67 \%$ for long-term predictions (39). For COVID-19 forecasting in China, LSTM was also used, and in comparison with the dynamic SEIR model, LSTM achieved promising results (43). Results showed LSTM achieved good forecasting performance because of its capacity to handle timedependent datasets. Ismail et al. modeled data from Denmark, Belgium, Germany, France, the United Kingdom, Finland, Switzerland, and Turkey using LSTM among several methods. They stated that LSTM is the most accurate model in comparison with the two other investigated algorithms, and they provided LSTM in order to make predictions in a 14-day perspective (44). This model is able to make realistic estimates based on the current situation and predict accurately the number of confirmed and recovered cases. Chandra et al. compared RNNs, LSTM networks, bidirectional LSTM networks, encoder-decoder LSTM networks, and convolutional neural networks (CNNs) with focus on univariate time series for multi-step-ahead prediction. The results showed that the encoder-decoder LSTM network, in addition to bidirectional LSTM, provides the best performances for given time series problems (37).

By predicting and preventing epidemiological peaks, we could achieve a "flattened curve" of the spread of the disease in order to prevent such a rapid spread of disease that could lead to overloading of national health systems and their collapse (45). Owing to the scarcity of the official data available and many unknown parameters in COVID-19 epidemic spread, development, and control, most early published models were prone to over-fitting, or parameters were taken from literature/on the basis of restricted and less precise evidence. This results in ambiguous results, especially because many articles are published ahead of peer review. Therefore, the main objectives of this study are to:

- carefully collect, harmonize, and unify epidemiological data from reliable sources, state, regional, and local levels, and incorporate them into the proposed models,

- investigate and compare two approaches in modeling the COVID-19 epidemiology, the SEIRD model and the deep learning model based on LSTM networks,

- train and test the investigated models on the cases in Benelux countries.

\section{MATERIALS AND METHODS}

We propose two main approaches, a standard compartmental epidemiological SEIRD model and a deep learning model based on LSTM networks, to describe the spread of COVID-19. From the aspects of disease progression notations, it should be emphasized that notation "severe cases" is equivalent to "hospitalized cases," and "critical cases" is equivalent to "ICU cases" in our article. However, for the training of the LSTM model, we have used the original data with the terms infected, hospitalized, and ICU; and for the SEIRD model, taking into account its definition, we have used subsequently derived terms mild, severe, and critical. The term deceased is a common term for both models.

The processing hardware included 64 GB of RAM, an NVIDIA Quadro RTX 6000 GPU, and an Intel(R) Xeon(R) Gold, 6240R, CPU running at $2.40 \mathrm{GHz}$. Tensorflow and Keras were used to implement the network in the Python 3.7.4, using the Spyder 3.3.6 environment.

\section{Compartmental SEIRD Epidemiological Model}

The basic structure of the SEIRD model is inspired by a number of studies on the natural clinical progression of COVID-19 infection (46). The choice for compartments to be used in the model depends on the features of the individual disease being modeled and the intent of the model. Passively immune class $\mathrm{M}$ and latent period class $\mathrm{E}$ are often ignored, because they are not essential to susceptible-infective experiences (3). The SEIRD model should more accurately reflect the progression of the epidemic than the simpler SIRD model that does not include an incubation period. The model used in this study is given in Figure 1. Although the standard SEIRD model has been used, to our knowledge, no previous models have included the division of infected class into three subclasses, mild, severe, and critical. Proposed SEIRD models, therefore, use a standard definition of compartments with the extension of modeling three infection stages. In addition, we have extended our model with a transmission rate mitigation factor to simulate the introduction of a variety of social measures (lockdown, etc.), mainly referring to the methodology introduced by Bastos et al. (47) and Morato et al. (48).

This yields to differential equations for the definition of disease spread:

$$
\dot{S}=-(1-\psi) \cdot\left(\beta_{1} I_{1}+\beta_{2} I_{2}+\beta_{3} I_{3}\right) S
$$

where $S$ represents the susceptible individuals who are infected. We have adapted the classical SEIRD model to include the effect of isolation by including $\psi(t)$, which represents a transmission rate mitigation factor. This factor expresses the observed social isolation ratio within the susceptible population. This means that $\psi=0$ is the case where there is no control over viral load (no social measures introduced), while $\psi=1$ represents a complete lockdown, without any social interactions (47).

Disease spread in the exposed (E) community is further described as

$$
\dot{E}=(1-\psi) \cdot\left(\beta_{1} I_{1}+\beta_{2} I_{2}+\beta_{3} I_{3}\right) S-a E
$$

The rate of development from the exposed stage to the infected stage $I$, where the patient becomes symptomatic and contagious, occurs at a pace $a$. Medical details of the various phases of 


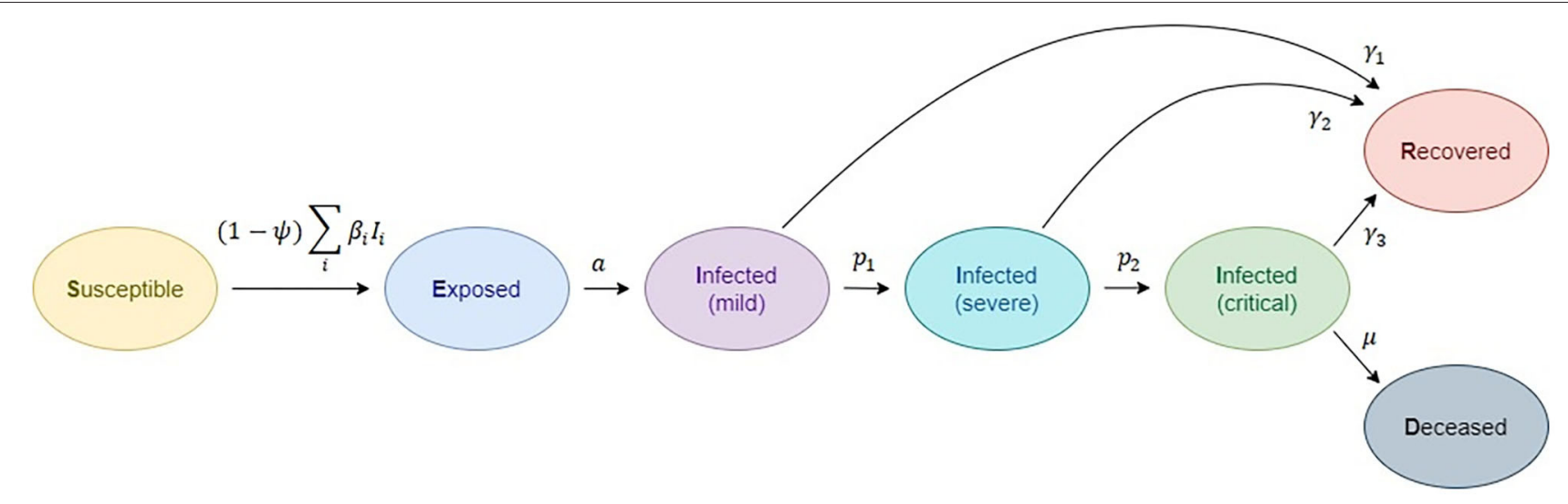

FIGURE 1 | Compartmental epidemiological model, based on the classic susceptible, exposed, infected (1- mild, 2-severe, 3-critical), recovered, deceased (SEIRD) model.

infection are given below. Infected individuals begin with mild infection $\left(I_{1}\right)$,

$$
\dot{I}_{1}=a E-\left(\gamma_{1}+p_{1}\right) I_{1}
$$

from which they either recover, at the rate of $\gamma_{1}$ or advance to severe infection $\left(I_{2}\right)$ at the rate of $p_{1}$ :

$$
\dot{I}_{2}=p_{1} I_{1}-\left(\gamma_{2}+p_{2}\right) I_{2}
$$

Severe infection subjects recover at rate $\gamma_{2}$ or progresses to critical stage $\left(I_{3}\right)$ at $p_{2}$ rate:

$$
\dot{I_{3}}=p_{2} I_{2}-\left(\gamma_{3}+\mu\right) I_{3}
$$

Recovered persons are defined by $R$ class/compartment and are supposed to be safe from re-infection for life:

$$
\dot{R}=\gamma_{1} I_{1}+\gamma_{2} I_{2}+\gamma_{3} I_{3}
$$

Individuals with critical infection recover at a rate of $\gamma_{3}$ and die at a rate of $\mu$.

$$
\dot{D}=\mu I_{3}
$$

Individuals can transmit the infection at any point, although at different levels. The transmission rate at stage $i$ is defined by $\beta_{1}$. This means that the used notation is: $S$ : susceptible individuals; $E$ : exposed individuals, infected but not yet infectious or symptomatic; $I_{i}$ : infected individuals in severity class $\mathrm{i}$, where severity increases with the increase of $i$, and we assume individuals must pass through all previous classes; $I_{1}$ : mild infection; $I_{2}$ : severe infection; $I_{3}$ : critical infection; $R$ : individuals who have recovered from disease and are now immune; $D$ : deceased individuals. Total population size is assumed to be constant in the form:

$$
N=S+E+I_{1}+I_{2}+I_{3}+R+D
$$

In order to describe the rates of disease progression from one category to another (susceptible to infected, infected to recovered or deceased, etc.), we use the following notation:

- $\beta_{i}$ rate at which infected individuals in class $I_{i}$ contact susceptible and infect them,

- $a$ rate of progression from the exposed to infected class,

- $\gamma_{i}$ rate at which infected individuals in class $I_{i}$ recover from disease and become immune,

- $p_{i}$ rate at which infected individuals in class $I_{i}$ progress to class $I_{i+1}$ and

- $\mu$ death rate for individuals in the critical condition.

The effects of lockdowns are, therefore, introduced, but due to the complexity of modeling that includes heterogeneity of policies in lockdown and mobility contacts, we have decided to decompose the modeling into shorter periods with respect to one consistent lockdown measure, and perform independent modeling on each of the sub-periods. In this study, we have adopted the value of $\psi$ based on the social measures of each of the investigated countries.

For Belgium (49), on March 12t 2020 , the Belgian federal government announced multiple far-reaching measures to flatten the curve of COVID-19 cases. The first measures included obligatory weekend closures of restaurants, bars, and nightclubs, as well as any non-essential stores until the end of the month. Schools at all levels were forced to close as well. Belgium went into its first lockdown on March 17. Non-essential shops had to close completely, working from home became the normal method of operation, and any non-essential movement or travel was forbidden. On March 20, national borders were mostly blocked, and most border traffic was halted. Despite the decision of the government to implement an exit plan, i.e., gradually loosening the restrictive restrictions, the measures remained in effect until the beginning of May. Expectedly, infection rates rose again, and by the end of July, the proclamation of new measures became urgent. From October 16 onward, restrictions were introduced again. A second full-fledged lockdown was in place until the end of November. Based on this, we have defined a table of 
TABLE 1 | Adopted values for transmission rate mitigation factor based on social measures (Belgium).

\begin{tabular}{lc}
\hline Time period & $\boldsymbol{\psi}(\boldsymbol{t})$ \\
\hline Before March 17th 2020 & 0 \\
March 17th-May 31st 2020 & 0.9 \\
June 1st-31st July2020 & 0.7 \\
July 31st-October 16th 2020 & 0.4 \\
October 16th-December 31st 2020 & 0.8 \\
December 31st 2020-March 15th 2021 & 0.7 \\
\hline
\end{tabular}

time-dependent factor $\psi(t)$ (Table 1). We have also incorporated a delay effect on the social distancing measures. The values are adopted based on the definition of finitely parametrized social distancing measures defined by Morato et al. (48). We stress that values of transmission rate mitigation factor should be taken only as guidelines that do not affect the conceptual essence of this study, since the proposed methodology is general and can be applied with respect to the epidemic reality of any location and population.

For Netherlands, after the introduction of lockdown at the end of March, there was a visible decrease in the number of infected people after this period. Strict measures were extended until May 3, and public events were banned until August. Measures were loosened at the beginning of August, resulting in another peak (although smaller than the first one, that was a result of no measures). This peak was also correctly predicted using the SEIRD model. It should be emphasized that the model is able to predict both increase and decrease in the number of cases, however, we outline here only the first two predicted peaks, as peaks are of greatest concern. Results on RMSE are given in Table 4, taking into account modeling for the whole period March 15, 2020 to March 15, 2021.

In Luxembourg, the measures of lockdown were introduced similarly as in Belgium, and the first peak in the epidemic was a result of previous viral load. Lockdown easing and protective measures were gradually loosened with the opening of cinemas and intra-European borders form June 15. This resulted in another peak, which was correctly predicted using the SEIRD model. It should be emphasized that the model is able to predict both increase and decrease in the number of cases; however, we outline here only the first two predicted peaks, as peaks are of greatest concern. RMSE is given in Table 4, taking into account modeling for the whole period March 15, 2020 to March 15, 2021.

Time variables in the proposed model are adopted from the literature:

- Average incubation period (in days),

- Average duration of mild infections (in days),

- Average duration of hospitalization (time to recovery) for individuals with severe infection (in days),

- Average duration of ICU admission (until death or recovery) (in days).

It should be mentioned that the average duration of hospitalization and those of infective periods do not include the incubation period, meaning that the total number of days from exposure to recovery should be the sum of the incubation period and average duration of hospitalization. The aforementioned values of COVID-19 parameters adopted from the literature are given in Table 2.

When it comes to the evaluation of model coefficients that are compatible with current clinical evidence, we primarily calculated certain parameters from real data available for the countries of Benelux [Belgium (67), the Netherlands (68), and Luxembourg (69)]. We focus on these countries because of the fact that the data were available in a tabular format with division into infection categories (ICU patients, hospitalized patients etc.). Not many countries provide data in such a format to the public. Available data for these countries that were important for calculating the parameters are: the number of infected patients, the number of hospitalized patients, the number of patients on ventilators, and the number of deaths. From these data, it is possible to calculate fraction of infections that eventually result in death, given as

$$
\text { case fatality rate }=\frac{\text { Total deceased cases }}{\text { Total infected cases }}
$$

In order to account for the delay with respect to the duration from the onset of infection to death, we have used adapted CFR (case fatality rate) based on definition from Shim (70). The denominator of the crude CFR formula includes infected persons whose fates are unknown, and those who have not yet died from the disease but will do so in the future. As a result of the time lag between infection and death, the CFR is skewed, a phenomenon known as right censoring (71). Statistical methods have been used to estimate the delay-adjusted CFR (70). The factor of adjustment, $u_{t}$, has been defined as

$$
u_{t}=\frac{\sum_{i=0}^{t} \sum_{j=0}^{\infty} c_{i}-{ }_{j} f_{j}}{\sum_{i=0}^{t} c_{i}}
$$

where $u_{t}$ represents the underestimation of the known outcomes and is used to scale the value of the cumulative number of cases in the denominator in the calculation of the CFR, $c_{t}$ is the daily case incidence at time $t$, and $f_{t}$ is the proportion of cases with a delay of $t$ from onset to death.

Also, it is possible to calculate the average fraction of infections that are mild, severe, and critical. People who are infected but not hospitalized have a mild infection defined as

$$
\text { mild infections }=1-\frac{\text { Total hospitalized cases }}{\text { Total infected cases }}
$$

where total hospitalized cases and total infected cases were taken from official reported data. Similar to adapting the CFR, delay was incorporated in the formulae given in equations 1113. Therefore, in equations 11-13, we also include the time of symptom onset to diagnosis, time of symptom onset to hospitalization, as well as hospitalization stay. Overall, the delay between symptom onset and hospitalization can be described by a truncated Weibull distribution with a shape parameter 0.845 
TABLE 2 | Adopted parameters for COVID-19 clinical progression based on literature sources.

\begin{tabular}{|c|c|}
\hline Parameter name & Adopted literature val \\
\hline Average incubation period, days & $5-6$ days; 5 days \\
\hline Average duration of mild infections, days & $7-12$ days; 5 days \\
\hline Average fraction of (symptomatic) infections that are mild & 80 \\
\hline Average fraction of (symptomatic) infections that are severe ${ }^{a}$ & 15 \\
\hline Average fraction of (symptomatic) infections that are critical ${ }^{a}$ & 5 \\
\hline Case fatality rate (fraction of infections that eventually result in death) ${ }^{a}$ & $2.27 \%$ \\
\hline Average duration of hospitalization (time to recovery) for individuals with severe infection, days & $5-14 ; 10$ days \\
\hline Average duration of ICU admission (until death or recovery), days & 14 days; $12-17$ days \\
\hline Reproduction number ${ }^{b}$ & $2-2.5 ; 2$ \\
\hline Infective period mild infection (in days) & 5 days \\
\hline Infective period severe infection (in days) & $7-12$ days \\
\hline Infective period critical infection (in days) & 14 days \\
\hline Transmission rate of mild infections & 0.4 per day \\
\hline
\end{tabular}

References

$$
(1,2,50-56)
$$$$
(50,57)
$$

As a default we assume that the dominant source of transmission comesfrom individuals with mild infections (e.g., $\beta_{1}>\beta_{2}>\beta_{3}$ ), who are likely to still be in the community, as opposed to isolated in the hospital.

a Parameters marked with this footnote are calculated based on available official data, and we did not use the literature data stated in Table 1. Values used are given in these table. ${ }^{b}$ The threshold for many epidemiological models is the specific reproduction number, RO, which is defined as the average number of secondary infections created when an infectious organism is introduced into a host population where everyone is susceptible. In many deterministic epidemiological models, infection will begin in a completely susceptible population only if an $R O>1$ is present.

TABLE 3 | Estimated parameters for COVID-19 clinical progression based on real data.

\begin{tabular}{lccc}
\hline Parameter name & \multicolumn{3}{c}{ Estimated value } \\
\cline { 2 - 4 } & Belgium (\%) & Netherlands & Luxembourg \\
\hline $\begin{array}{l}\text { Average fraction of } \\
\text { (symptomatic) infections } \\
\text { that are mild }\end{array}$ & 95.96 & 96.89 & 98.65 \\
$\begin{array}{l}\text { Average fraction of } \\
\text { (symptomatic) infections } \\
\text { that are severe }\end{array}$ & 4.04 & 3.11 & 1.35 \\
$\begin{array}{l}\text { Average fraction of } \\
\text { (symptomatic) infections } \\
\text { that are critical }\end{array}$ & 0.86 & 1.05 & 0.2 \\
\hline
\end{tabular}

and a scale parameter 5.506. The methodology was based on (72). Severe infection refers to individuals who are hospitalized but not on ventilators, and critical infection refers to those on ventilators, both of which are defined as

$$
\begin{gathered}
\text { Total hospitalized cases }- \text { total cases on ventilators } \\
\text { Total infected cases } \\
\text { critical infections }=\frac{\text { Total cases on ventilators }}{\text { Total infected cases }}
\end{gathered}
$$

Based on the described methodology, parameters calculated for Belgium, Netherlands, and Luxembourg are given in Table 3.
TABLE 4 | Root mean square error between simulated and official statistical data for Belgium, Netherlands and Luxembourg using SEIRD model.

\begin{tabular}{llll}
\hline RMSE & Belgium & Netherlands & Luxembourg \\
\hline & mean \pm std & mean \pm std & mean \pm std \\
\hline $\begin{array}{l}\text { Mild infected } \\
\begin{array}{l}\text { Severe infected } \\
\text { (hospitalized) }\end{array}\end{array}$ & $\begin{array}{l}\text { 240.79 } \pm 90.556 \pm 2.64 \\
\text { Critical infected (ICU) }\end{array}$ & $219.52 \pm 20.49$ & $198.54 \pm 78.53$ \\
deceased & $0.58 \pm 0.49$ & $0.05 \pm 0.002$ & $0.15 \pm 0.1$ \\
\hline
\end{tabular}

\section{LSTM Epidemiological Model}

Since the course of the epidemic is rapidly changing and the mathematical SEIRD models have some limitations due to the nature of partial differential equations, we implemented an algorithm that is suitable for data fitting and forecasting based on time-series data. For the problems of this category, adequate architecture will be based on recurrent neural networks (RNNs). The specificity of RNNs is reflected in the context layer, whose purpose is to act as memory in order to merge the current state and inputs for the propagation of information into latter states. A method for training RNNs is backpropagation through time (BPTT), which is a kind of extension of the backpropagation algorithm. BPTT is characterized by a gradient descent where the error is backpropagated for a deeper network architecture that contains time-defined states. For this reason, there is the 
problem of learning long-term dependencies, which will lead to vanishing and exploding gradients. In order to address the vanishing gradient problem of RNNs, long short-term memory (LSTM) neural network was developed. LSTM is a special kind of recurrent neural network structure that can comparatively learn the proposed long-term dependencies and overcome the mentioned drawbacks of RNNs. LSTM contains memory cells and gates that provide better capabilities in remembering the long-term dependencies. An LSTM cell was introduced by Hochreiter and Schmidhuber (73). Compared with the RNN memory cell, the LSTM memory cell has two components to its state: the hidden state and the internal cell state.

The LSTM cell consists of the gates shown in Figure 2, the input gate $I_{t}$ decides which information can be transferred to the cell, then forgets gate $f_{t}$ decides which information from the previously cell should be neglected. The control gate $\bar{C}_{t}$ is controlling controls the update of the cell, and the output gate $\mathrm{O}_{t}$ controls the flow of output activation information.

As it is shown in Figure 2, LSTM calculates hidden layer $H_{t}$ as

$$
\begin{aligned}
I_{t} & =\sigma\left(W^{i} \times\left(X_{t}+H_{t-1}\right)+b_{i}\right) \\
f_{t} & =\sigma\left(W^{f} \times\left(X_{t}+H_{t-1}\right)+b_{f}\right) \\
O_{t} & =\sigma\left(W^{o} \times\left(X_{t}+H_{t-1}\right)+b_{o}\right) \\
\bar{C}_{t} & =\tanh \left(W^{c} \times\left(X_{t}+H_{t-1}\right)+b_{C}\right) \\
C_{t} & =\sigma\left(f_{t} * C_{t-1}+I_{t} * \bar{C}_{t}\right) \\
H_{t} & =\tanh \left(C_{t}\right) * O_{t}
\end{aligned}
$$

The number of the input features is presented as $X_{t}$, and $H_{t}$ is the number of hidden units. Learning started with the zero initial values of $\mathrm{C}_{0}$ and $\mathrm{H}_{0}$. Also, during the learning process, some parameters were adjusted, such as bias given as $b$ and weight given as $W$. The internal memory of the unit is given as $C_{t}$, and it should be emphasized that all the gates have the same dimension as the size of your hidden state (74).

The encoder-decoder LSTM (ED-LSTM) network was developed as a sequence-to-sequence neural network to effectively map a fixed-length input to a fixed-length output. The advantage of these neural networks is that the mentioned two lengths of inputs and outputs do not have to be the same. For that reason, this neural network achieved state-of-the-art results in the field of automatic text translation. The ED-LSTM network has two implementation phases: the first phase, encoding, and the second phase, decoding. The purpose of the first phase is to encode an input sequence into a fixed-length vector representation and compute a sequence of hidden states, and the purpose of the second phase is to decode the vector representation and define a distribution of the output sequence. The architecture of the ED-LSTM is presented in Figure 3.

\section{RESULTS AND DISCUSSION}

In this section, we present the results of the applied SEIRD model and LSTM encoder-decoder model, and compare them with the real situation of the COVID-19 outbreak and spread in Benelux countries. Data were monitored during the 1-year period, from March 15, 2020 to March 15, 2021. Values for the numbers of people infected, hospitalized, in ICU as well as deceased were taken from Infectious Diseases Data Explorations \& Visualizations (68) for Belgium (67) for Netherlands, and (69) for Luxembourg.

\section{Results for the SEIRD Model}

The results for the SERID model showed that the model is effective at predicting several peaks of the epidemiology. Due to the fact that transmission rate mitigation factor was included in the model to simulate the social measures (no isolation to complete lockdown), the model is able to catch more than one peak, but also captures the fall in the trend of epidemiological situation. It should be emphasized that predicted numbers represent current numbers (daily predictions) of the infected cases (mild, severe, critical), but the number of deceased cases is cumulative.

Figure 4 shows the comparison between the official data and simulated curves for mild cases in the aforementioned characteristic sub-periods. The match between the trends of the real epidemiological situation and predicted progress shows that the model is able to follow the course of epidemiology, whether there is an increase or decrease in the number of infections.

Figure 5 shows the comparison between the official data and simulated curves for severe cases in the aforementioned characteristic sub-periods. The match between the trends of the real epidemiological situation and predicted progress here is even better than for the mild cases.

Figure 6 shows the comparison between the official data and simulated curves for critical cases in the aforementioned characteristic sub-periods. The difference between the trends and peak positions of the real epidemiological situation and predicted values is small, with an almost perfect fit.

Figure 7 shows the comparison between the official data and simulated curves for deceased cases in the aforementioned characteristic sub-periods. The difference between the trends and peak positions of the real epidemiological situation and predicted deceased values is small, with an almost perfect fit.

As it can be seen, tracking the number of people with severe infection, critical infection, or deceased people shows a very good match. The trend and peak value of predicted curves show a promising match for all the monitored curves, mild, severe, critical, and deceased. Some differences can be observed in peak positions for some sub-periods between the simulated and real cases. This is may be due to the initial conditions set in simulation, or adopted values of certain parameters from literature, which can be further optimized. Nevertheless, the trend is adequate, showing that the methodology can be used to describe the epidemiology.

The same methodology was performed for all the three countries (Belgium, Netherlands, and Luxembourg), as well as investigated groups (mild, severe, critical, and deceased). We only present the complete figures for Belgium to demonstrate the methodology, while for Netherlands and Luxembourg, we only show the match for peaks, as they are of greatest interest.

Figure 8 shows the comparison between the official reported data and simulated curves. Regarding the comparison of official 


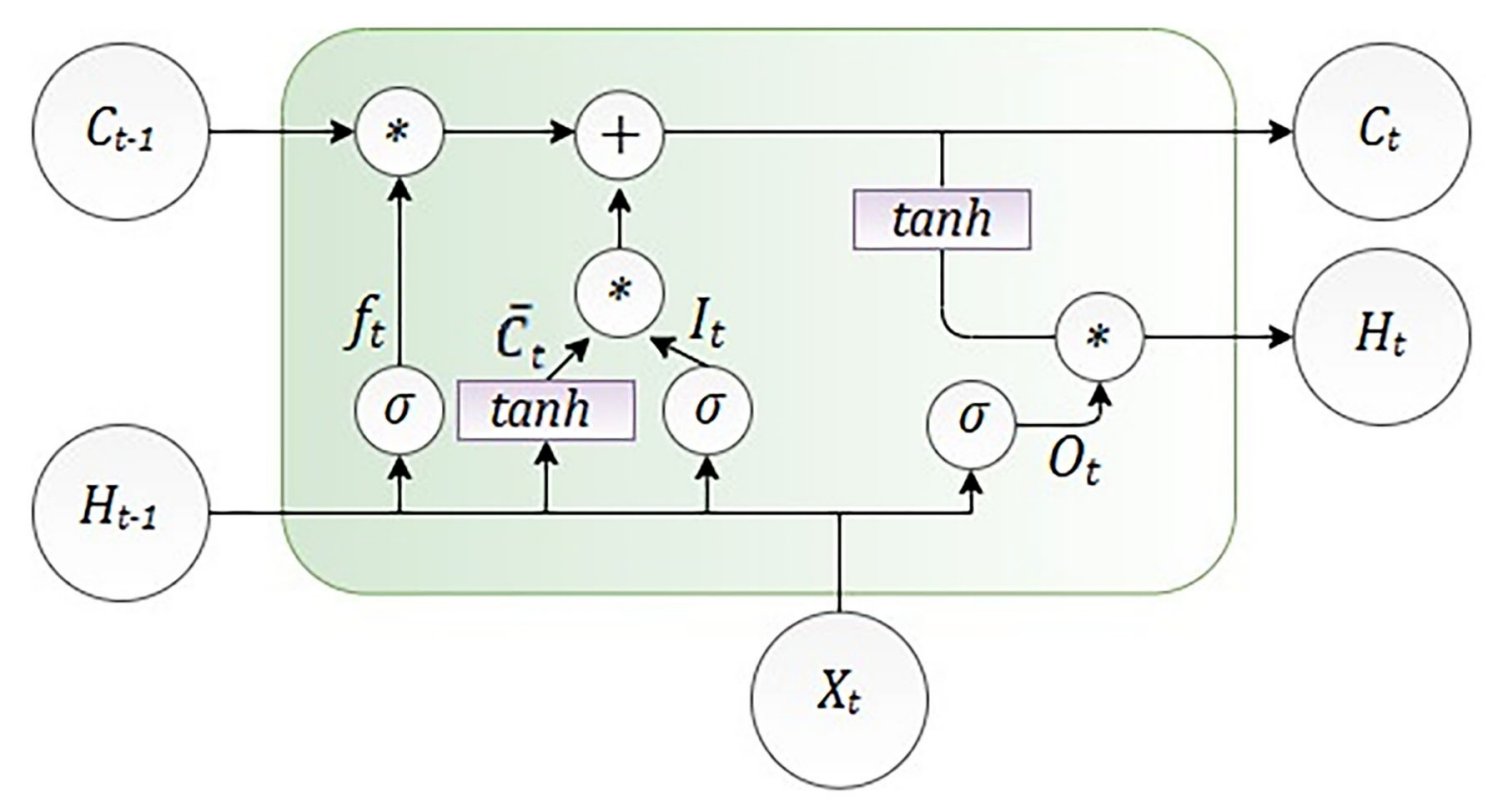

FIGURE 2 | Architecture of one long short-term memory (LSTM) cell.

statistical and simulated values, for the case of Netherlands, the trend in all four classes, infected critical, infected severe, infected mild, and deceased are matching, as well as the position of the peaks. It can be seen that in the cases of infected people with a severe or critical condition, as well as deceased people, the curves match perfectly with very small deviations between simulated and official data.

For the case of Luxembourg, it can be seen that the trend and fluctuations are matching for number of simulated and real number of people with mild infections. For classes severe and critical infections, as well as deceased, the trends and values of the predicted simulation line match well with the official reports (Figure 9).

Table 4 shows the root mean square error (RMSE) between simulated and real values of investigated curves in time for Belgium, Netherlands, and Luxembourg. The values are given as mean \pm standard deviation, because of the fact that we have calculated RMSE for each modeled subperiod. We applied the moving average smoothing technique to the real values in order to remove the fluctuations between days and calculated RMSE between the smoothed curve and simulated curve. The results show very small RMSE for all three countries and classes of infection. The only larger value of RMSE is for mild infections, which can lie in the fact that mild cases were derived based on other data (total cases and hospitalized cases), as there are no available data on specific mild category.

All this shows that there are many factors involved in the prediction of the epidemic spread, and that more factors can be taken into account in order to account for decreasing and increasing trends, etc. However, if looking at shorter periods of time and modeling on such subperiods, number of deceased and infected people (mild, severe, and critical), using official statistics and simulated numbers shows good match, which means that the models is showing promising results and can be further upgraded to take into account different underlying complex phenomena.

In comparison with the existing literature, Bastos et al. (47) proposed an adapted susceptible-infected-recovered (SIR) model, for the purpose of incorporating under-reporting in Brazil and the response of the population to confinement measures, widespread use of masks, etc. They discuss that the most optimal method for epidemiological modeling should be based on recurrent model tweaking (through identification), with the uncertainty margins always taken into consideration (47). Morato et al. (48) also investigated modeling using the SIRD model, which includes time-varying auto-regressive immunological parameters in the case of Brazil (48). Their main contribution is adding analytical regressions, least-squares optimization, and auto-regressive model fits. Köhler et al. (75) studied adaptive techniques for resilient and optimum management of the COVID-19 pandemic using social distancing measures and applied them in the case of Germany (75). Using the SIDARTHE model, they introduced key features that distinguish between detected and undetected cases, symptomatic and asymptomatic individuals, with the additional separate state for patients with life-threatening symptoms. Similarly, in the case of Germany, Kantner et al. (76) proposed adjustments to traditional SEIR models by introducing the optimal solution computed by minimizing the terminal cost function. Alleman et al. proposed a discrete model predictive controller for optimal government response to the COVID-19 outbreak that would not result in overloading the number of ICU beds in Belgium (77). Their method mostly focused on calibration of the social interaction parameter, which results 


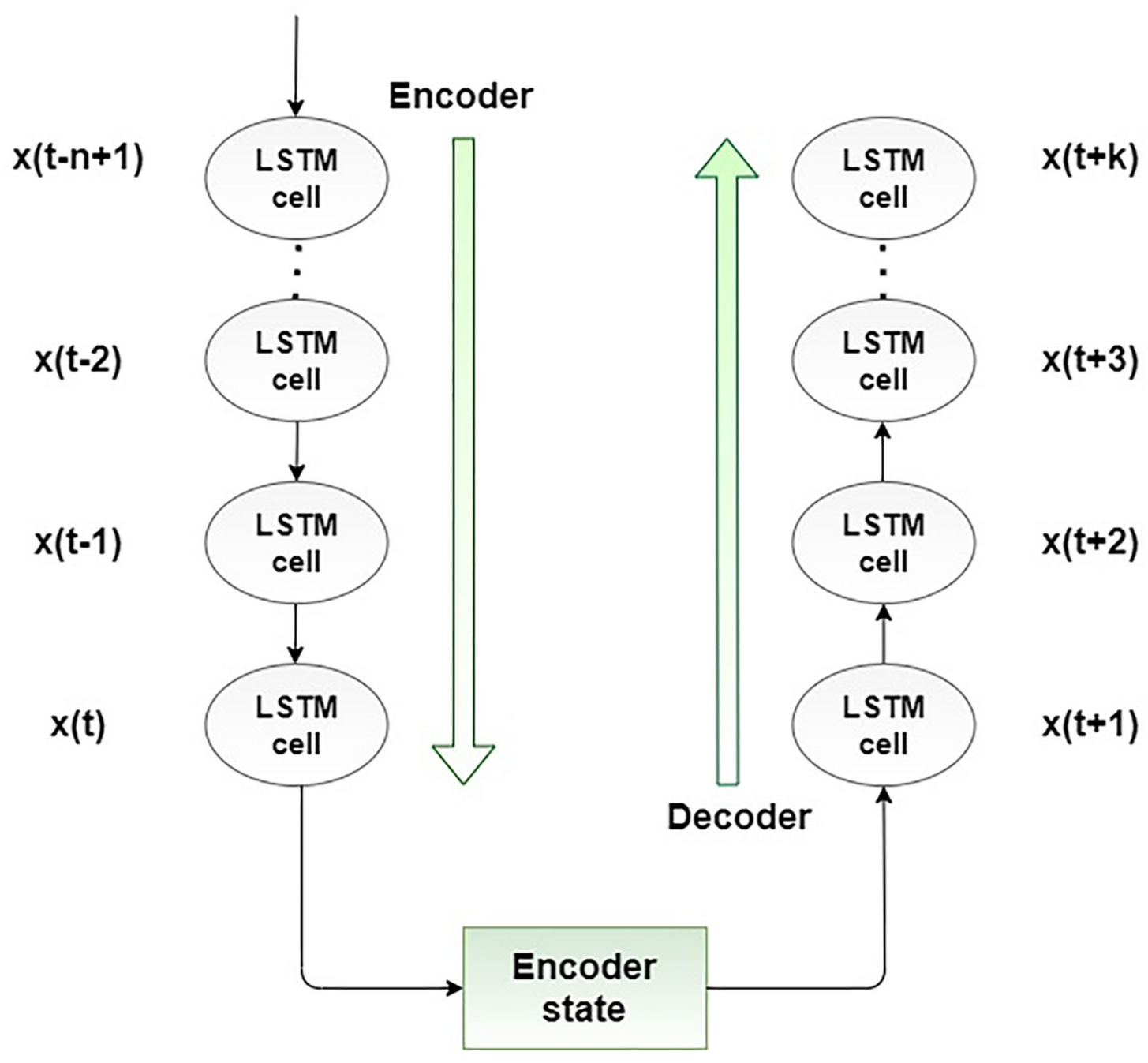

FIGURE 3 | Proposed encoder-decoder LSTM structure.

in an improved model in comparison with the state-of-the art models.

Our model introduces a traditional SEIRD model but with improved methodology, classification of infected class into three subclasses, mild, severe, and critical. It also takes into account the transmission rate mitigation factor as a form of modeling social measures (partial to full lockdown etc.). Time-related parameters are considered with time delays. RMSE is competitive with presented state-of the results, with space for additional improvements. The added value of this study lies in the validation of the proposed methodology using official data from national authorities in Benelux countries. Despite the fact that some aspects of epidemics are taken into account, there are certain simplifications of the model. The main limitations of the SEIRD model may come from different aspects:

- values of parameters estimated based on official statistics are not correct, and official statistics are scarce or not reported accurately: it has already been reported in other articles that official statistics have underreported the real numbers in the beginning, leading to the fact that initial conditions were not taken correctly and, therefore, later simulated, and real numbers were different. A predictive model by Imai et al. used travel volumes from Wuhan and used the dates when imported cases first arrived in cities within China to forecast the size of the epidemic in Wuhan (78). They reported that substantially more cases were present in Wuhan than were reported in the official statistics (79). The same conclusion was met in an article by Korolev (80). He asserts that even though the fraction of all cases that are reported are not identified, it can be effective to consider the underreporting. If it is assumed that all cases are reported and the estimates of $\mathrm{R}_{0}$ are based only on that, the value of $\mathrm{R}_{0}$ may be biased downward. It may lead to overestimating the number of deaths (80). 


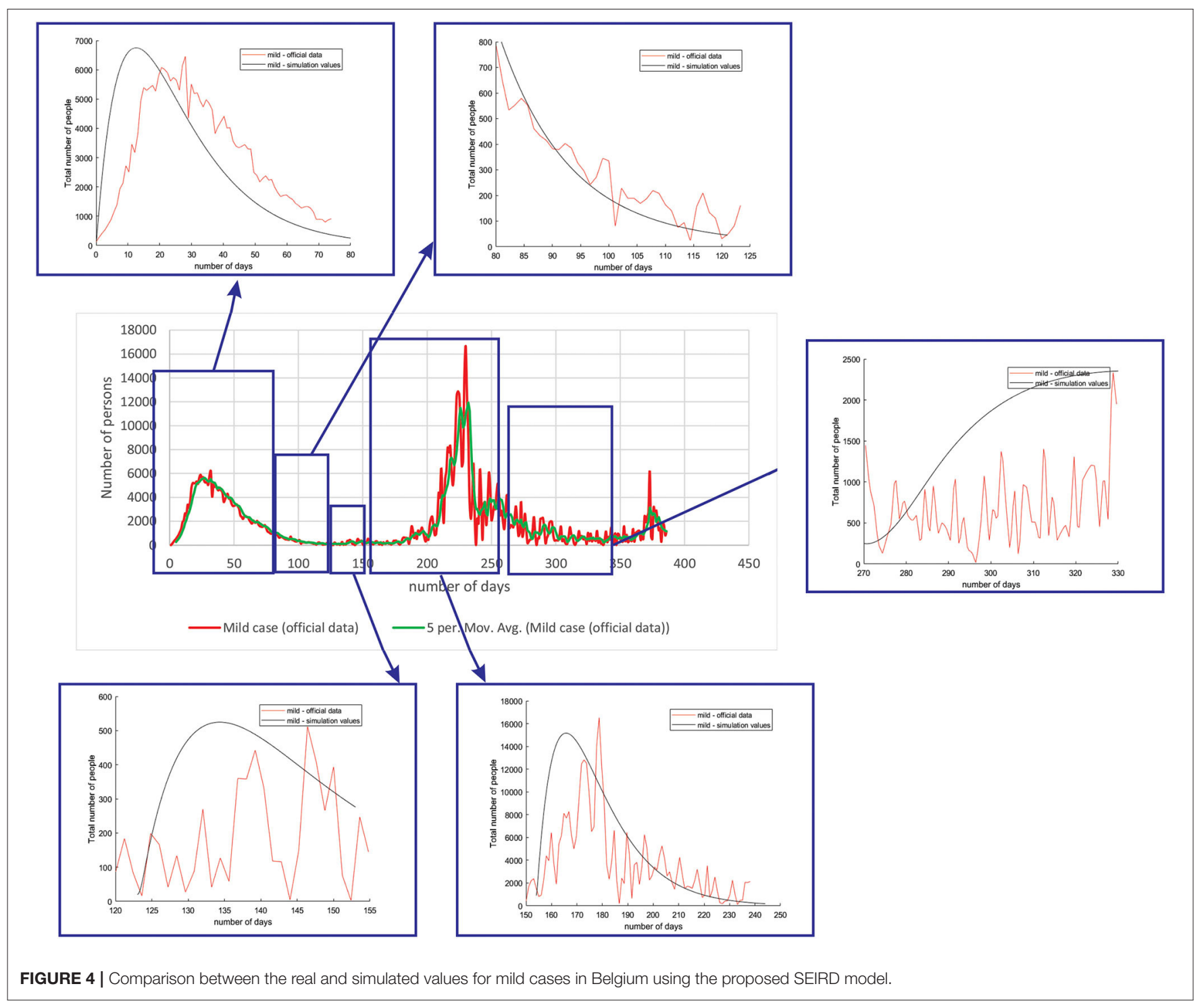

- the complexity of the COVID-19 epidemic spread and development is yet to be determined, and the current SEIRD model does not take into account medical interventions, number of hospital beds available, etc. The real case is much more complex, with many other additional phenomena included (behavioral responses to the epidemic, re-infection, no immunization, mutations of the virus, variants of concern, mobility of people, etc.)

Although the model is showing promising results, and the match between the curves between the simulated trends and values calculated based on officially available data are well-matching, there are some differences in peak values and positions. This can primarily be due to the fact that modeling the spread of the disease is complex and includes many phenomena, out of which only several main are included in the current model. Therefore, the main limitation of this study is limited number of phenomena modeled (no reinfection, asymptomatic infection, medical intervention, etc.). Therefore, we have investigated models based on deep learning and further report the results for one such LSTM-based model.

\section{Results for the LSTM Encoder-Decoder Neural Network}

Unlike the mathematical model where the number of exposed, susceptible, infected, and deceased were simulated, for the LSTM-ED model, the focus is on univariate time-series data of daily infected, hospitalized (but not in ICU), patients in ICU, and deceased cases. We have used Tensorflow and Keras to implement the neural network in Python 3.7.4. The Keras function for the LSTM layer has an argument called the initial state that includes a list of initial state tensors to be transferred to the first cell call. We set this argument to the default value that involves zero-filled initial state tensors. We have adopted 


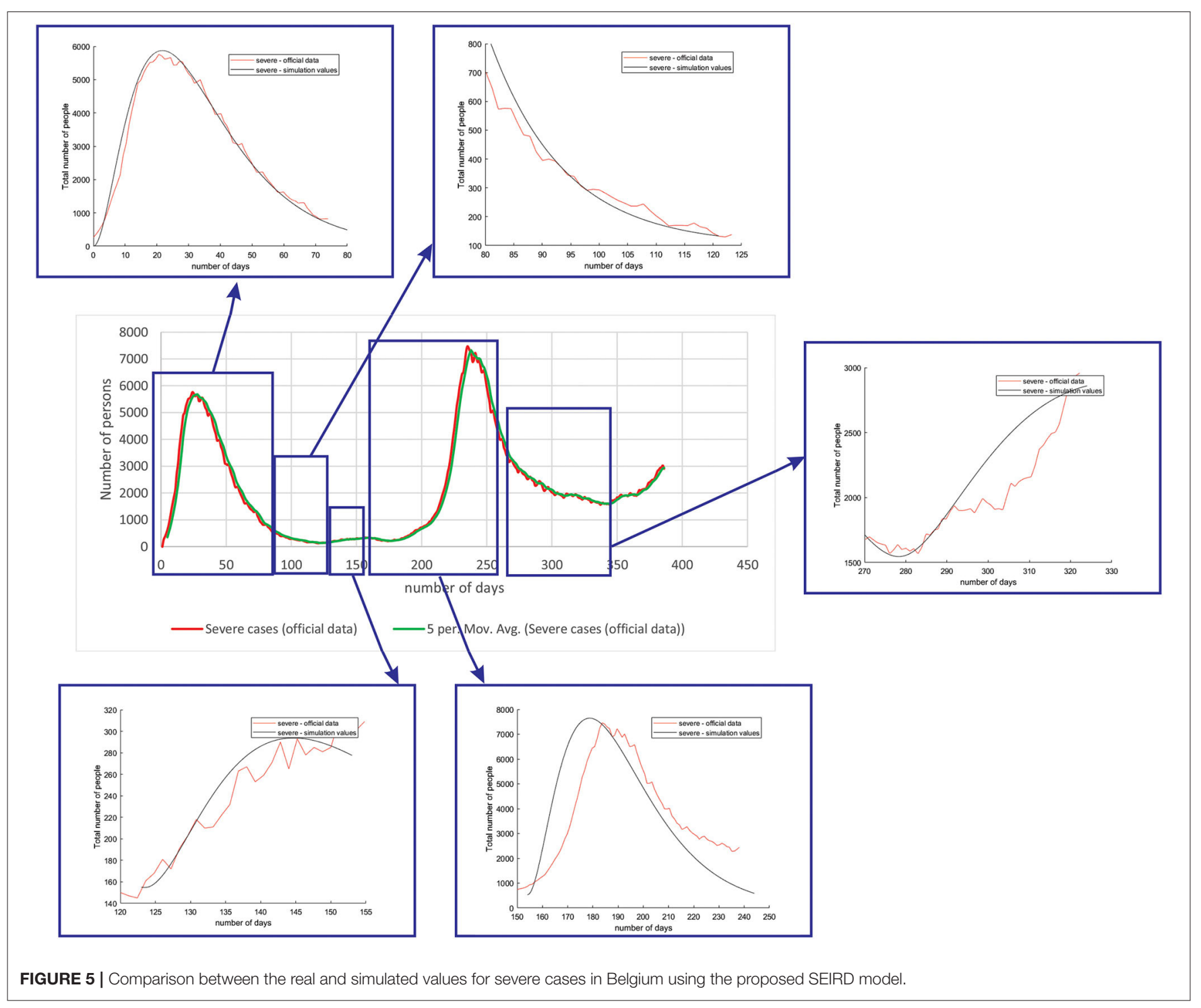

grid search method for hyperparameter optimization, and we iterate a different number of nodes that have been used in the hidden layer, dropout rate, optimizer, epochs, and batch size. The best number of neuron units in hidden layers is set to be 60 , with a dropout rate of 0.4. As optimizer, Adam has been selected and batch size to be 32 . The model has been trained during 100 epochs. We used the cross-validation process in order to get a more realistic view of the error and predictions of the model. It means that for the first iteration, we trained the LSTM-ED model with the 50-day dataset from March 15, 2020 to May 3, 2020 , then we used the trained model to forecast each variable of the 50-day test dataset from May 4, 2020 to June 22, 2020. The mentioned process is repeated in additional four iterations. The second iteration implies training data from the beginning, March 15, 2020 to June 22, 2020, and the test data from June 23, 2020 to August 12, 2020. The third iteration implies training data up to August 12, 2020 and test data from August 132020 to October 2, 2020. It is clear that the training data in each subsequent case will include all the data that were analyzed in the previous iterations, including both the training and test sets of the previous iterations. The test dataset in the fourth iteration contains 62 samples instead of 50, and it means that data from October 3, 2020 to December 4, 2020 are included. The last iteration implies the training data from March 15, 2020 to December 4, 2020, and the test data from December 5, 2020 to March 15, 2021. In this case, the training set is larger than in previous iterations; accordingly, the test set is also larger and includes 100 days. The example of cross-validation process and mentioned iterations is presented in Figure 10. In the following example, the number of hospitalized cases in Belgium was used as input data.

In order to estimate the error between real and predicted values, mean squared error (MSE) has been set as a loss function. Loss function during the training process is shown in 


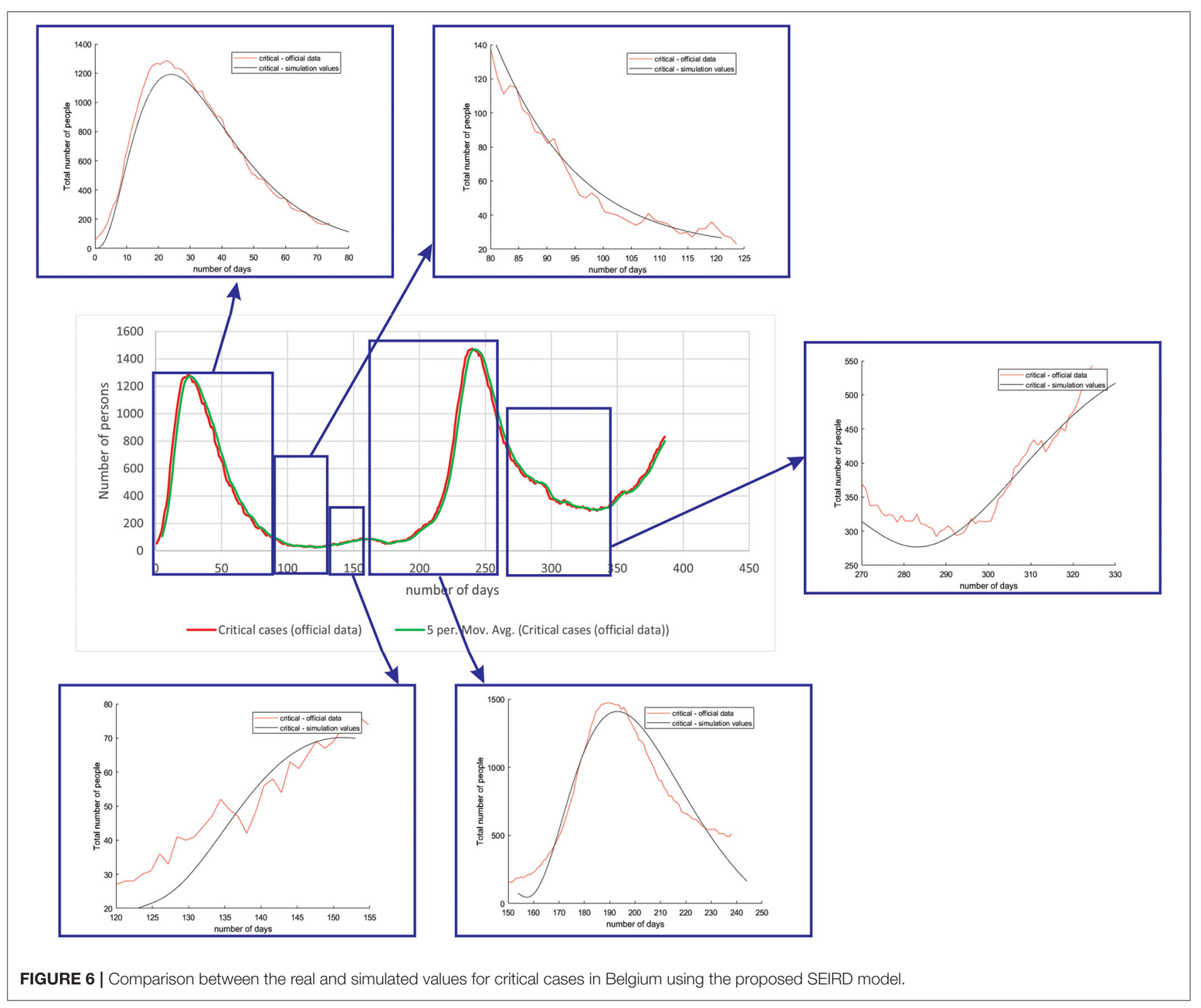

Figure 11. Loss function is presented in each iteration of the cross-validation process.

As evaluation metrics, root MSE (RMSE) mean absolute error (MAE) and R2 score are used. The average values of the metrics of all the five iterations for all the three countries is given in Table 5. Due to large fluctuations in the real data, we compared the forecasted curve with the smoothed version of the real curve. We applied the moving average smoothing technique to real values of the test dataset in order to remove the variation between time steps. Actually, we created a new series where the values comprised the average of 5 days of observations from the real data.

During the validation process, we concluded that LSTM-ED is capable of predicting when another peak of the epidemic will occur, based on the position of the first peak. Therefore, we decided to show for each variable individually how the network will forecast the values for $\sim 100$ days. In the case of Belgium, in this 1-year period, only two peaks appeared, so the period October 20, 2020 to March 15, 2021 was used for the testing dataset. This means that the dataset is divided in the following manner: $58 \%$ for training and $42 \%$ for the testing process. The comparison between the official statistical data and simulated curves is shown in Figure 12. Also, a smoothed curve is presented in order to remove a noise and expose better a trend of the official data curve. The trend of the predicted curve matches well the smoothed curve from official data for all the monitored curves, infected, hospitalized, ICU, and deceased. For the case of Netherlands and Luxembourg, we made a different division and returned to the original decision to take a test set consisting of 100 days, which includes the data from December 6, 2020 to March 15, 2021.

In Figure 13, comparison between the official statistical data of Netherlands and simulated curves is shown. It can be seen that in the cases of infected and deceased people, the trend is 


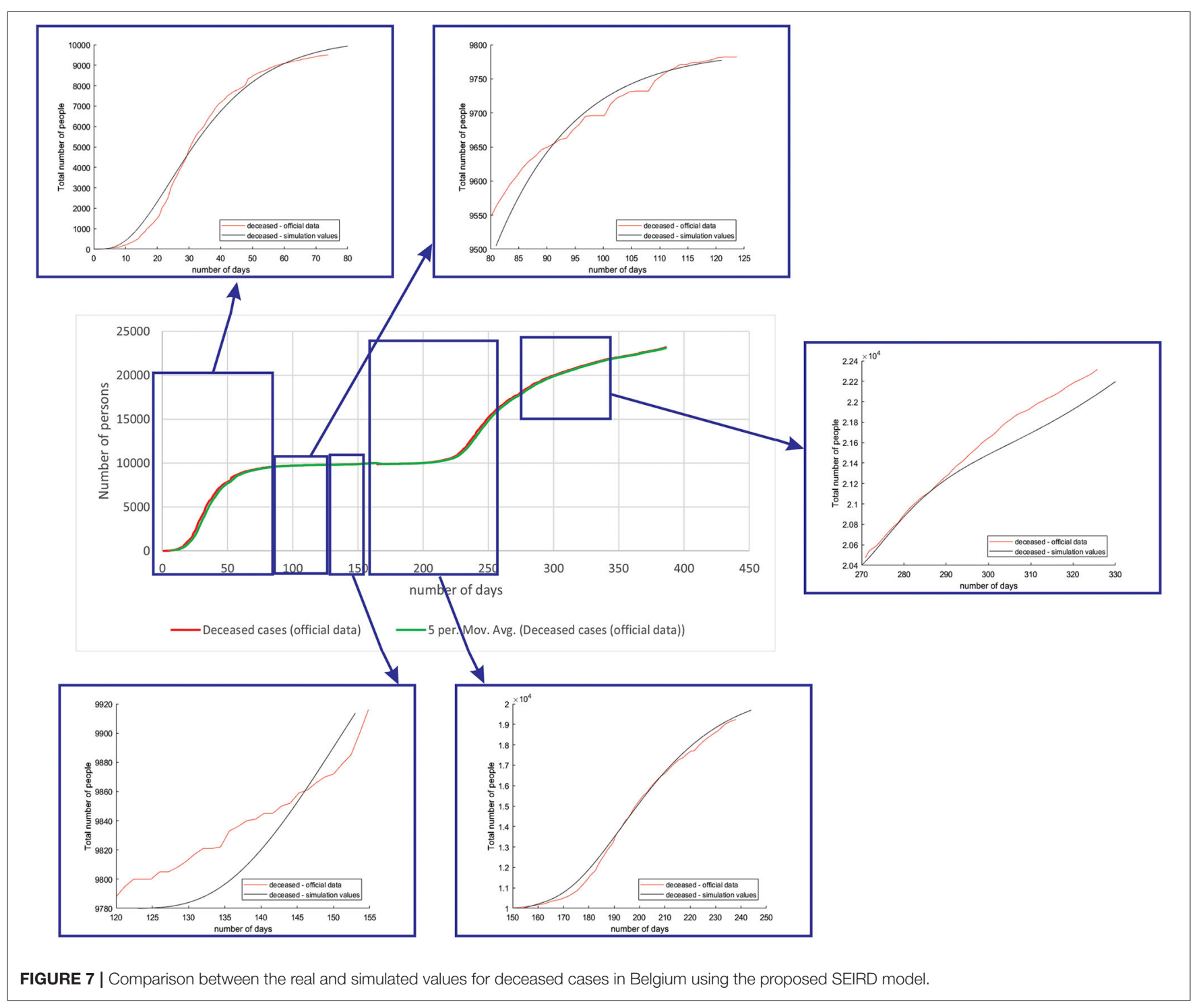

matching with very small deviations between official statistical and smoothed curves, as well as position of peaks. However, for the cases of hospitalized and ICU, the position of peaks is matching, but the height of the peaks differs a little more than in previous cases.

In Figure 14, comparison between the official statistical data of Luxembourg and simulated curves is shown. The trend of the predicted curve matches well the smoothed curve from official data for all the monitored curves, infected, hospitalized, ICU, and deceased. However, in the last days of the test set, the simulated curve tends to flatten, so we can conclude that soon there will be no peak like the highest peak of the epidemic in Luxembourg.

The aim of our study was to establish the architecture of the model and hyperparameter settings in order to forecast several COVID-19 categories. Our model is able to forecast number of infected patients per day, number of hospitalized patients per day, number of patients in intensive care units, number of patients with a fatal outcome. As far as we know, most of the literature has been oriented toward predicting only the total or cumulative number of infected (positive) cases per day. Vadyala et al. (81) proposed K-means-LSTM neural network to construct a prediction model for short-term forecasting of confirmed COVID-19 cases in Louisiana, United States (81). As a result, the proposed method achieved forecasting performance with an RMSE value of 601.2. Ayyoubzadeh et al. (82) used LSTM and linear regression models to estimate the number of positive COVID-19 cases in Iran. To evaluate the robustness of the model, 10-fold cross-validation was utilized, and, as performance evaluation criterion, RMSE was used. According to the experimental results, the LSTM model achieved an RMSE value of 27.187 (82). Furthermore, Wang et al. (41) proposed a model based on LSTM neural networks in terms of daily and cumulative forecasting of infected patients in Russia, Peru, and Iran (41). They stated that the existing forecasting 


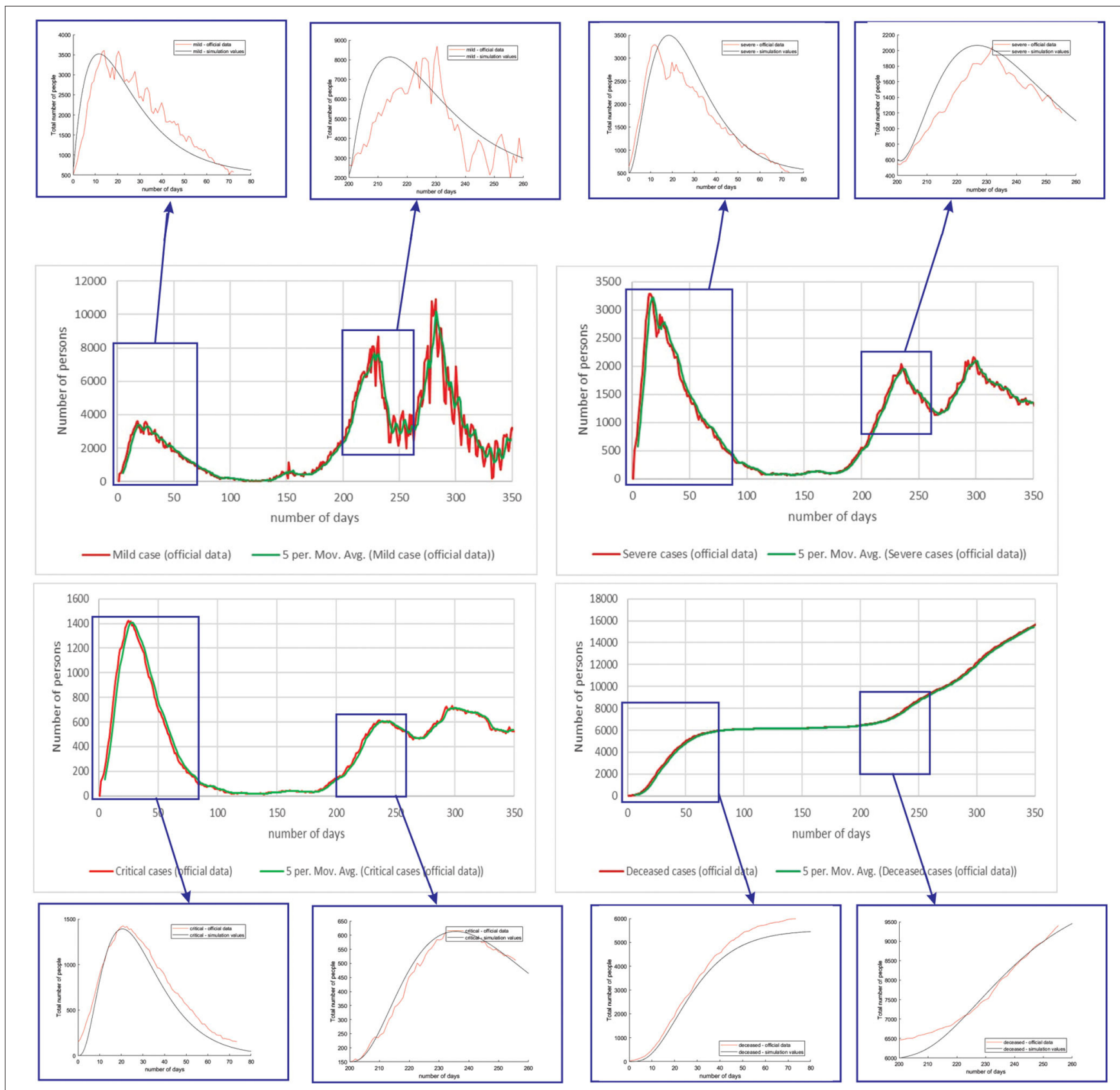

FIGURE 8 | Comparison of real and simulated values for the four categories of interest (Netherlands), mild infection, severe infection, critical infection, and deceased people.

LSTM model can only predict the epidemic trend within the next 30 days accurately, so they included an additional mechanism for the purpose of long-term forecasting. Taking into account the limitations of previous studies, the aim from the aspect of deep learning methodology was to use an encoderdecoder LSTM model for long-term forecasting of the spread of COVID-infections in the Benelux Union. We select the countries with a reliable source of COVID-19 statistics, because in these countries the situation with and without social measures was present, with the aim to establish a model that will be able to forecast in a long-term manner. Selection of different countries as the test set does not affect the conceptual essence of this study, since the proposed methodology is general and can be applied with respect to the epidemic reality of any location and population. Our results show that the LSTM model proves to be promising for long-term forecasting, so the established methodology can be applied for any country or region. 

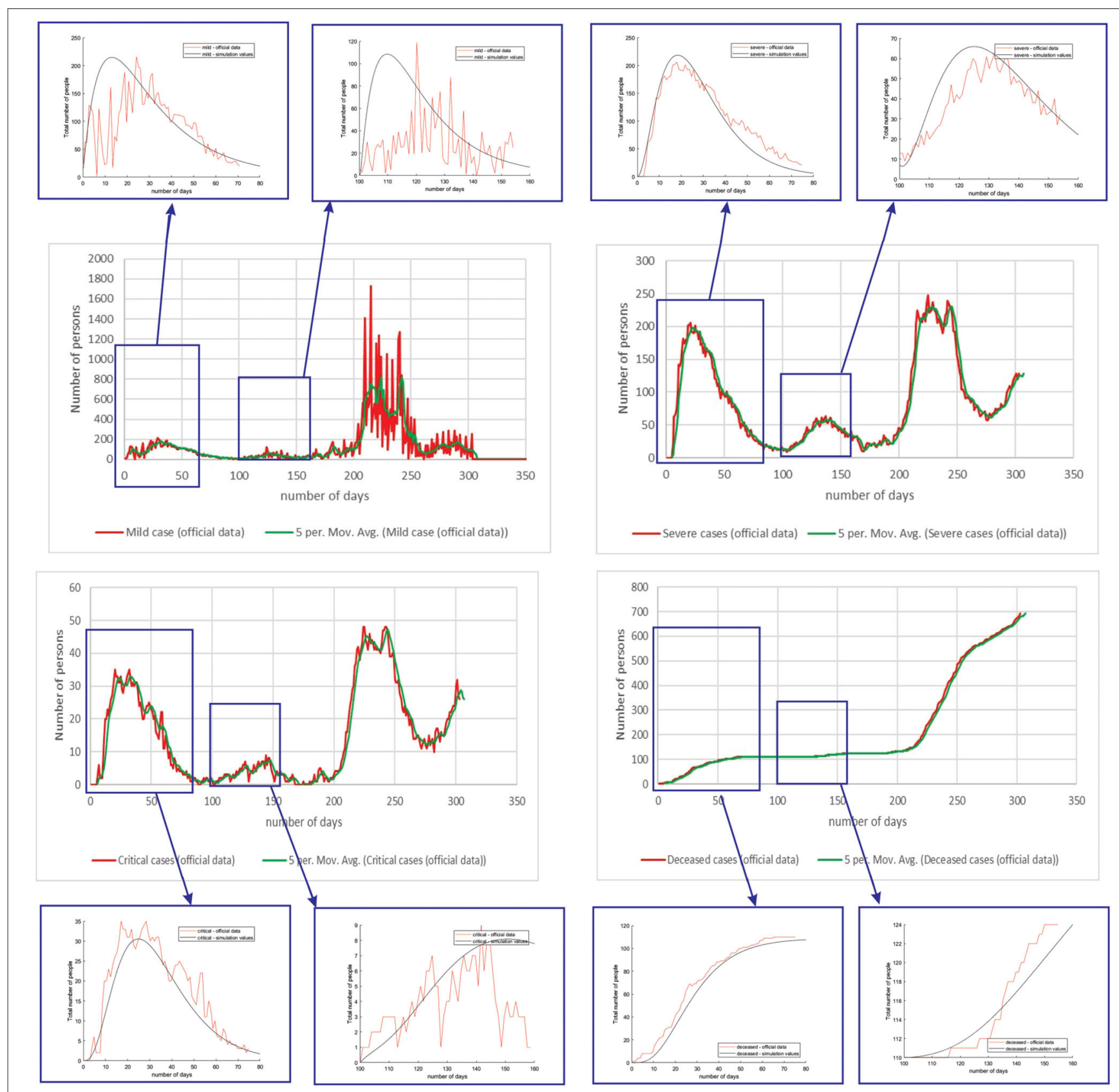

FIGURE 9 | Comparison of real and simulated values for the four categories of interest (Luxembourg), mild infection, severe infection, critical infection, and deceased people.

\section{Complex Phenomena to Be Included as Possible Extensions of the Model}

Modeling COVID-19 is very complex, and many phenomena are yet to be included in future models. Although the initial analyses from this study show promising results, some simplifications had to be adopted. We plan to address them in future:

- by the definition of the infected compartments, it is being assumed that asymptomatic cases are not infective. Although this is an idealized case, and many studies suggest that people infected with COVID-19 may be temporarily asymptomatic and infectious before developing symptoms, there are also studies that indicate that asymptomatic cases may not be infectious (83). Future expansion of the model will deal with infectious asymptomatic cases.

- stochastic formulation may be used to incorporate flexibility (uncertainty) in the predictions and obtain improved estimates of the parameters. In order to capture the stochastic nature of the transitions between the compartmental populations in such models, methods such as Markov Chain 

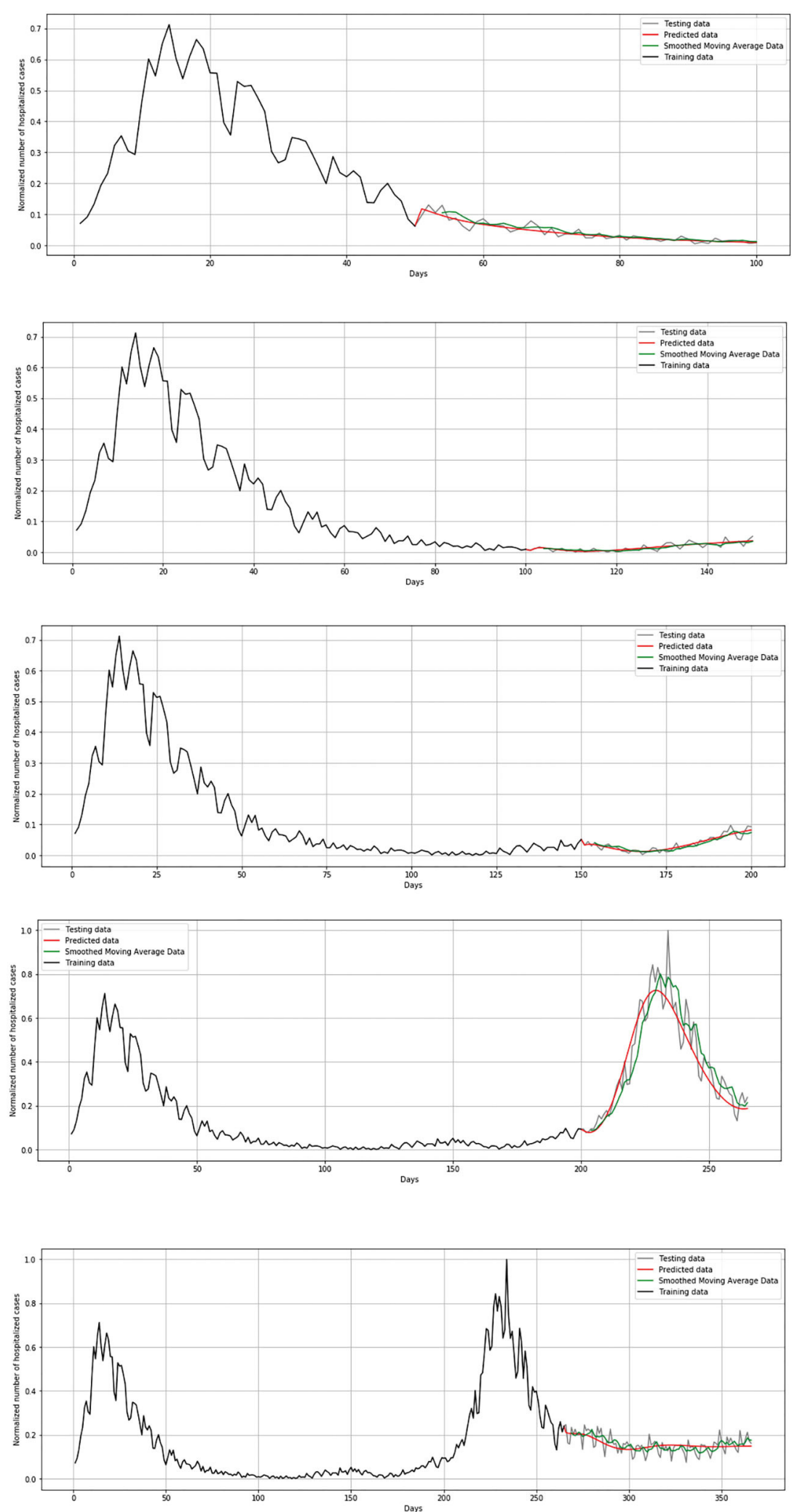

FIGURE 10 | Cross-validation process for hospitalized cases in Belgium. 
TABLE 5 | The regression metrics on test data of Belgium, Netherlands and Luxembourg using LSTM model.

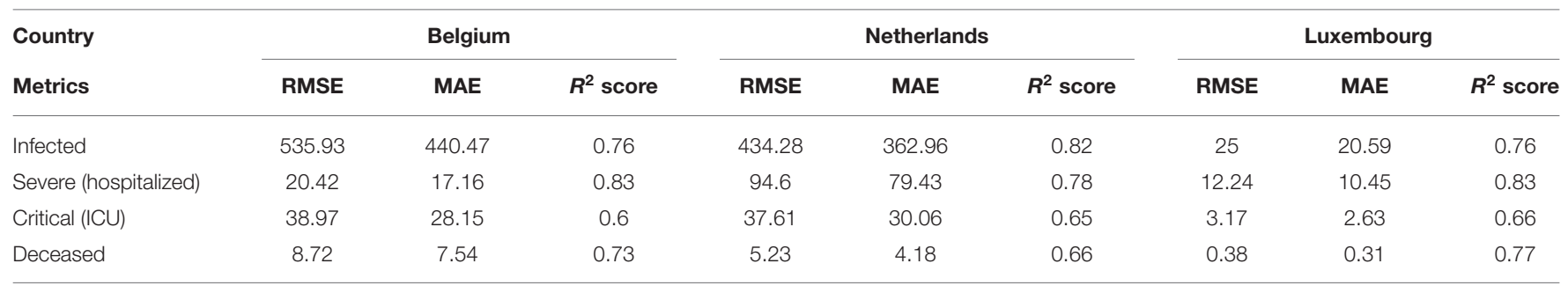

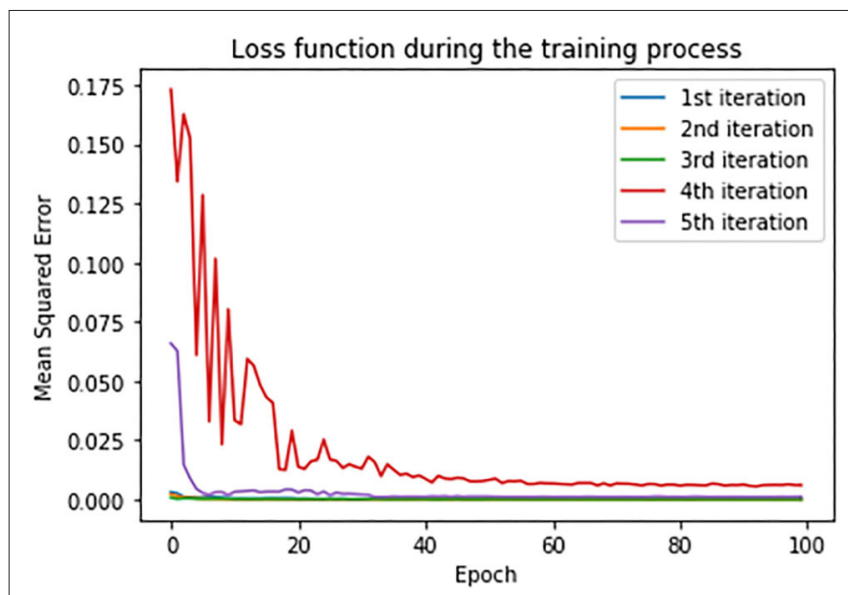

FIGURE 11 | Loss function in training set during the cross-validation process.

Monte Carlo can be used (84). Future discussion on improved model parameters will include the stochastic nature of epidemiological modeling.

- although LSTMs have proven efficient in terms of utilizing time-series data for prediction, there is a gap in the existing solutions that can combine both spatial and temporal aspects of the dynamics of COVID-19. Melin et al. proposed selforganizing neural networks and a fuzzy fractal approach in order to bridge this gap and address the issue of spatial variation (85). Although their results seem promising, the validation of results is only shown on 10 days prediction, which does not guarantee the accurate prediction of longer periods. Khan et al. (86) proposed a hybrid convolutional neural network (CNN) and long short-term memory (LSTM) model in order to extract multi time-scale features from convolutional layers of $\mathrm{CNN}$ and to learn short, medium, and long time series dependencies (86). Their approach seems promising, with results reported to be better in comparison with LSTM networks. This is also the direction we will investigate in the future.

- mobility-contact effects were not taken into account at this point of model validation. During the first week of new measures (starting on Friday, March 13) a steep drop in mobility between many cities has been noted. Mobility in March decreased to around 50\%, 60\% of January levels (87). From the perspective of neighboring countries, the border cities in Germany and in the Netherlands also noted less traffic, but the drop happened a little bit slower than in Belgium. In the border cities of Lille and Luxembourg, traffic is significantly reduced, at the highest level, among the considered regions. Furthermore, even though Dutch and German border cities noticed slightly higher inner-city mobility than the cities in Belgium, the drop in cross-border traffic decreased to around 50\%, 60\% of January level of traffic. If we consider Belgian inner-city traffic, the last week of March was characterized by an even more drastic reduction in overall mobility hovering just about $30 \%$ of January levels. Traffic between cities dropped between 10 and $20 \%$ of their January levels. If we take into account these statistics and information that Belgium tightened measures after March 17 and introduced complete lockdown, this can imply that cross-border mobility might not have a significant impact on the spread of the virus and worsening of the current situation in these countries. Different methods can be used to improve the validation and verification processes in order to find the parameters of the system dynamics model, meaning the parameters of the pandemic (88) discussed that in their study the effect of lockdowns can be tracked by a reduction in mobility reported by Google (89). In this study, we are focused on describing how the comparison between the standard SEIRD model and the LSTM model has achieved high accuracy results.

The SARS-CoV-2 virus already has hundreds of variations. Pathogen surveillance is performed for each country by the national authority. Although first identified on April 62021 in Belgium, the delta (B.1.617.2) variant of concern in August 2021 is dominant lineage in the country, accounting for more than 95\% of all the variants (90). According to preliminary findings, this variation is also related with greater transmissibility and quicker spread. For Netherlands, RIVM, The National Institute for Public Health and the Environment is undertaking laboratory research to determine which versions of the virus are present and what this means for the spread of the virus in the country. Out of the total number of samples, the most frequent variant is the alpha (B.1.1.7) variant, after which the delta (B.1.617.2) and beta (B.1.351) versions are less frequent (91). All the three variants have had an estimated reproduction number higher than that of the old variant of the virus. In Luxembourg, community surveillance showed that the delta (B.1.617.2) variant represents the dominant one (accounting for $99.1 \%$ ), with low prevalence of the gamma (P.1) variant (accounting for 0.9\%) (92). This major question of variants will help in terms of investigation 


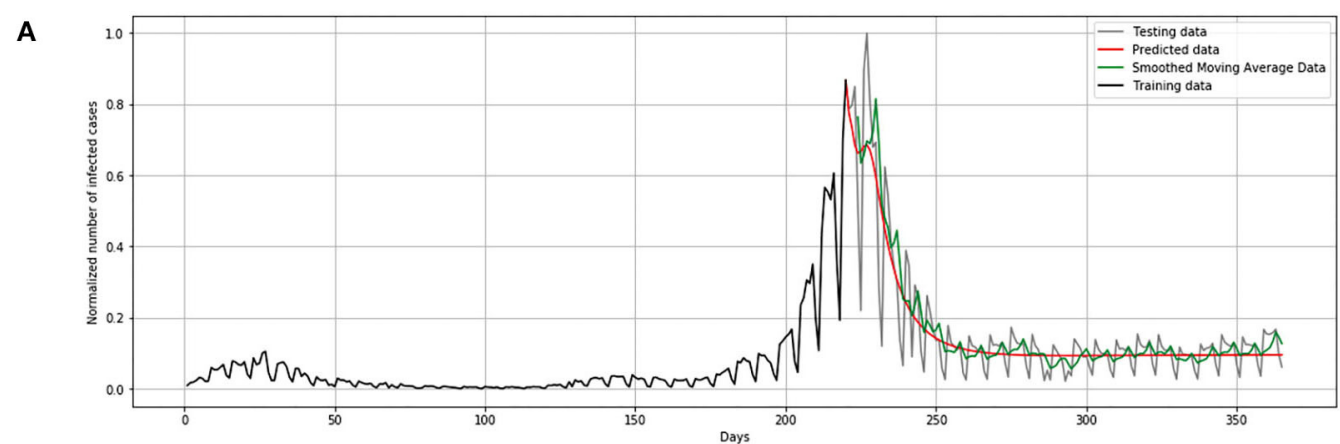

B

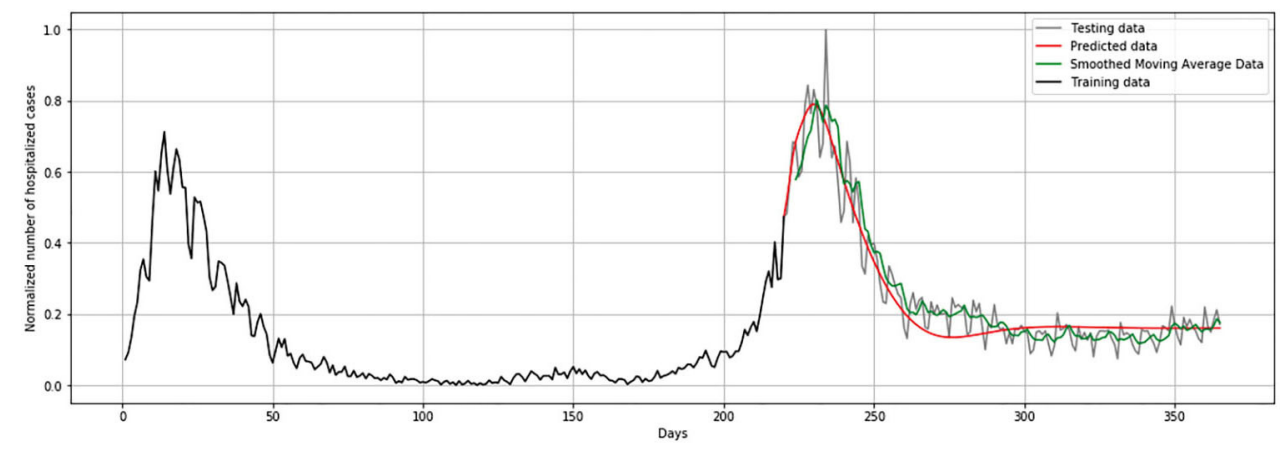

C

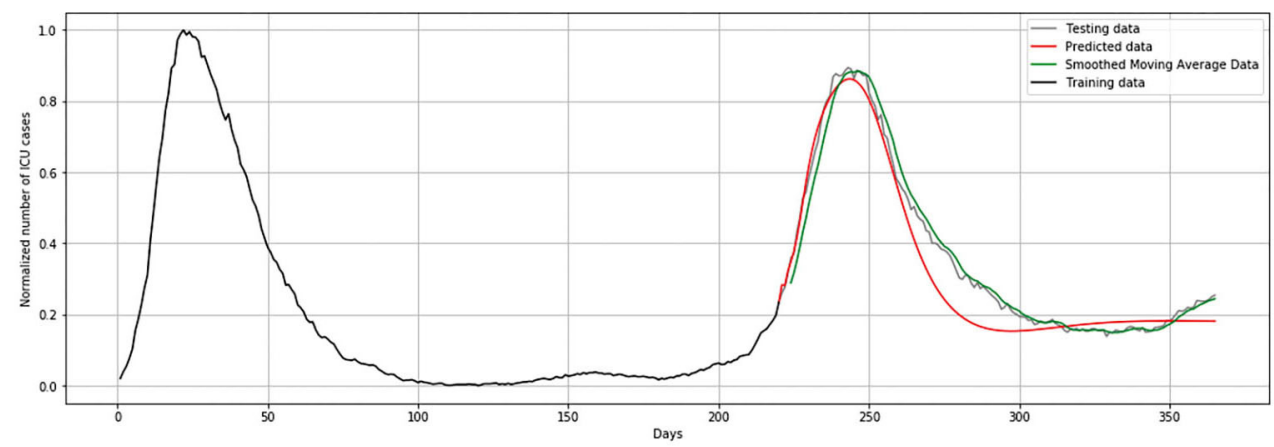

D

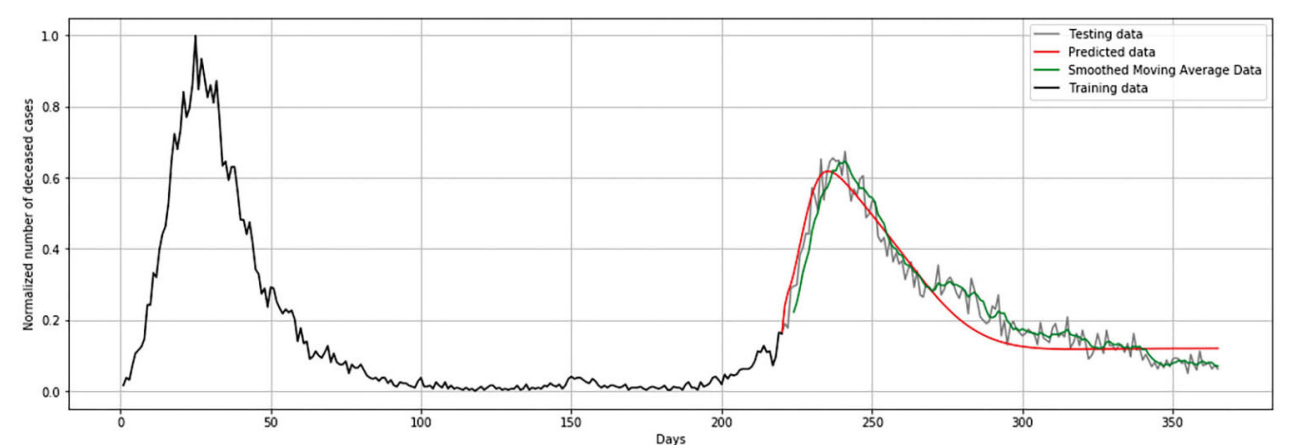

FIGURE 12 | Comparison of real and simulated values for Belgium: (A) number of infected cases, (B) number of hospitalized cases, (C) number of ICU cases, (D) number of deceased cases. 
A

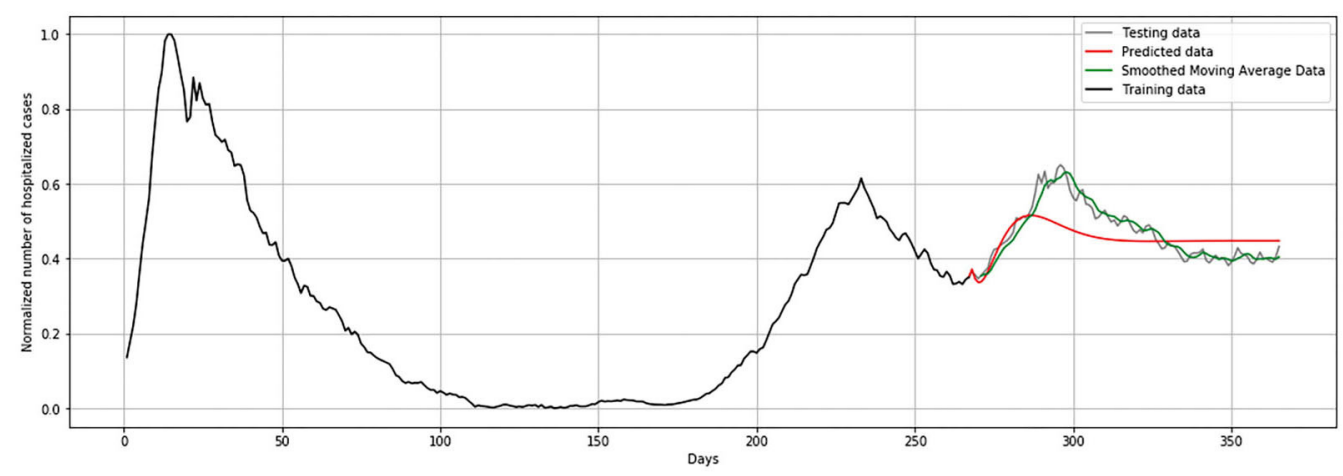

B

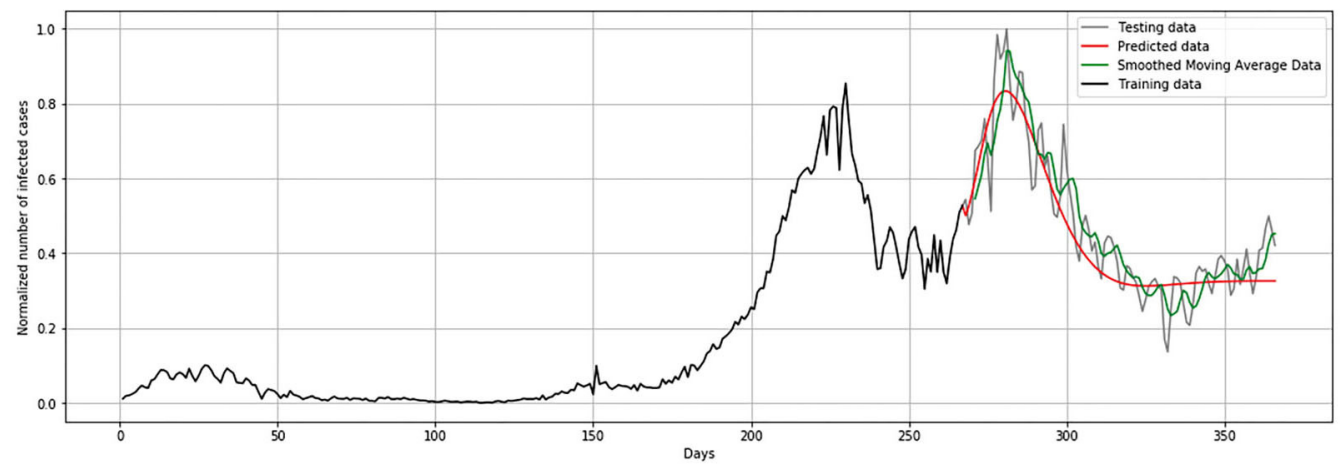

C

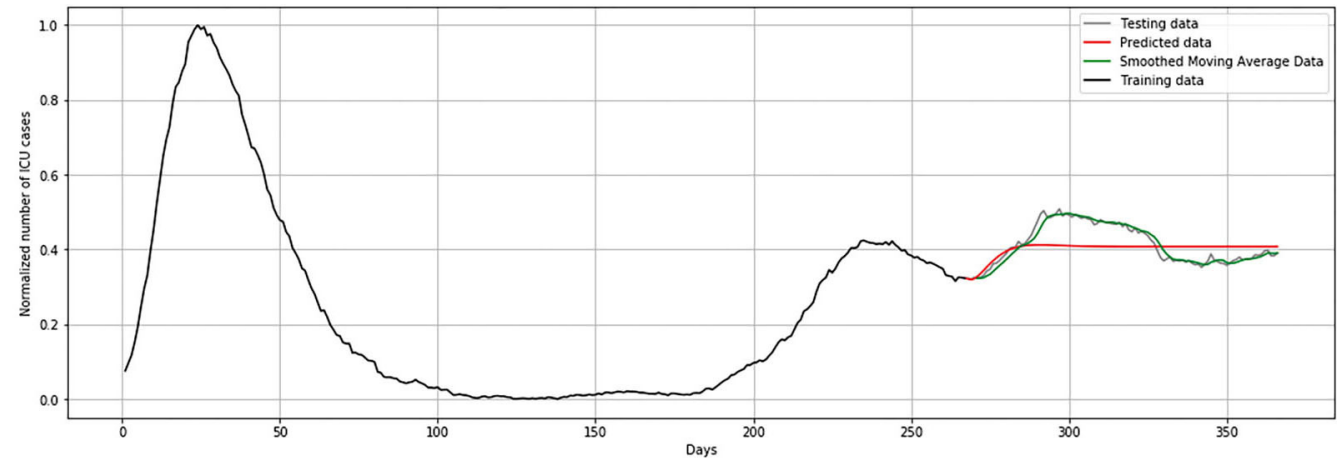

D

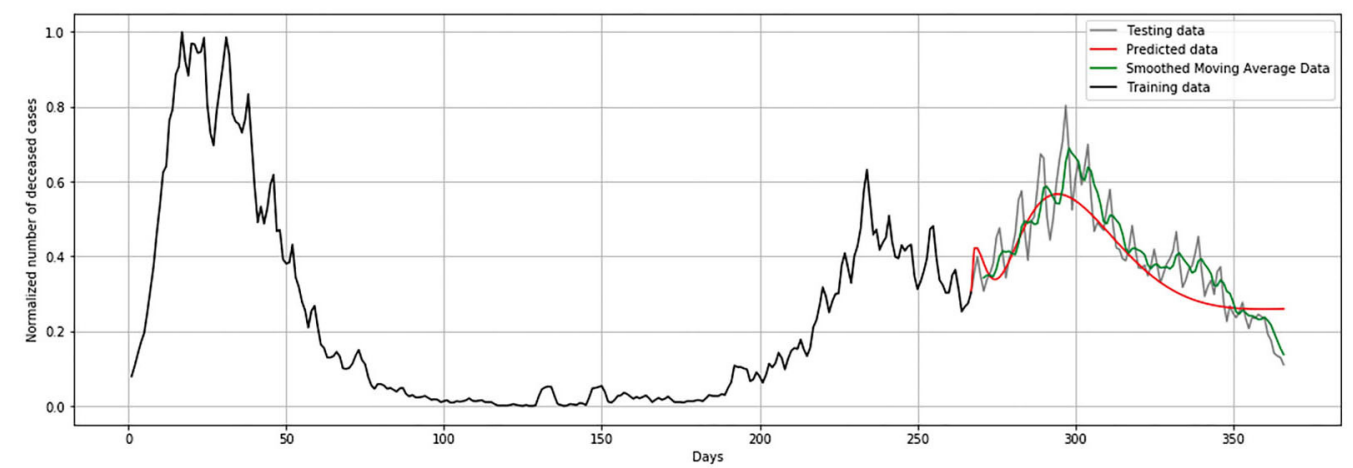

FIGURE 13 | Comparison of real and simulated values for Netherlands: (A) number of infected cases, (B) number of hospitalized cases, (C) number of ICU cases, (D) number of deceased cases. 
A

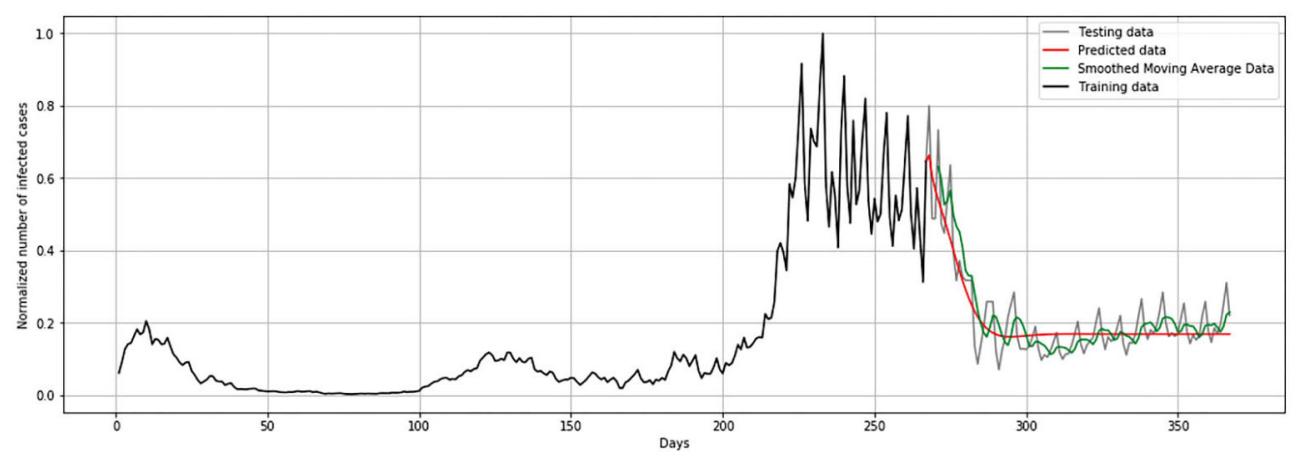

B

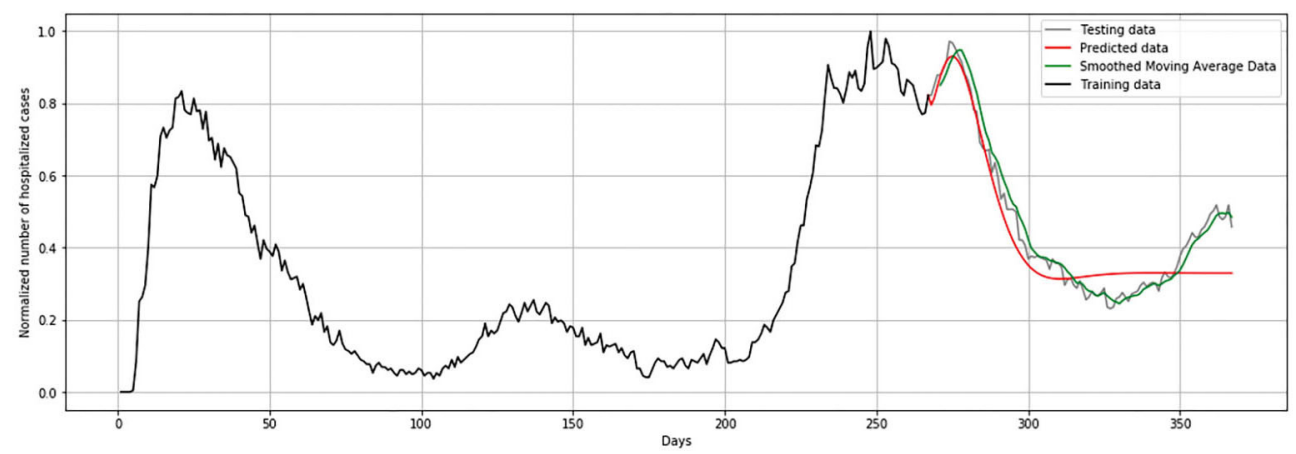

C

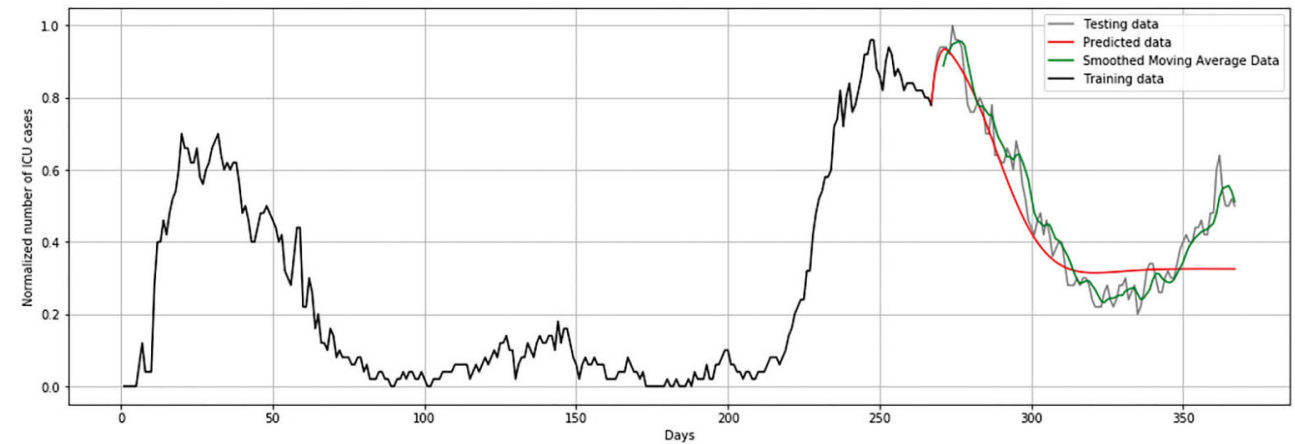

D

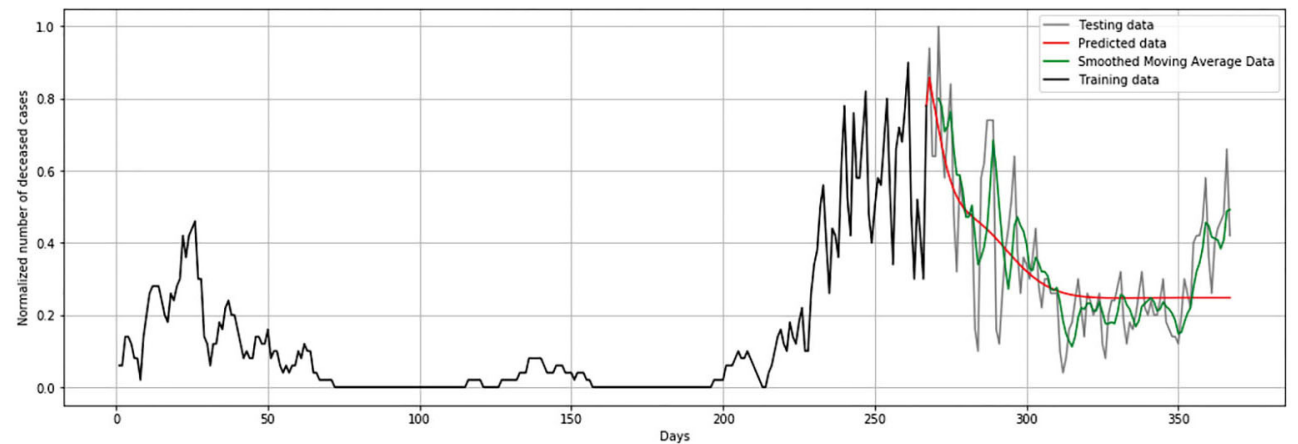

FIGURE 14 | Comparison of real and simulated values for Luxembourg: (A) number of infected cases, (B) number of hospitalized cases, (C) number of ICU cases, (D) number of deceased cases. 
whether they are more easily spread, cause more illness, or if viral variations do not react as well to immunization.

Additionally, at this point, there are very little publicly available epidemiological data per day on patients with COVID19 with respect to variants, especially on the level of the country. Results from genome-wide association studies (GWAS) in terms of trait-associated genetic variants can be used as control variables in epidemiology studies to account for confounding genetic group differences (93). The harmonized individual-level data of some participating cohorts from Belgium (BeLCovid_2), Brazil (BRACOVID), Italy (COVID19Host(a)ge_4, GEN-COVID), Spain (COVID19-Host(a)ge_1,2,3, INMUNGEN-CoV2, SPGRX), and Sweden (SweCovid) are under preparation to be deposited at the European Genomephenome Archive (EGA) (94). This will be a future direction for research and model update.

\section{CONCLUSIONS}

This study describes the modeling of COVID-19 spread and development in the population, using two proposed methodologies, the SEIRD model and the model based on LSTM neural networks. The COVID-19 epidemic was declared a pandemic by the WHO since the number of infected people grows exponentially, and many countries have decided to impose certain measures, such as a complete lockdown of affected cities to reduce the number of contacts and stop the spread of the virus. Our proposed method included the SEIRD compartmental epidemiological model with included components, susceptible, exposed, infected (we have divided the infected group into three subgroups, mild, severe, and critical), recovered, and deceased, with included effects of lockdown modeling. In order to calculate the parameters for the model, we have also investigated official statistical data for the countries of Benelux (Belgium, Netherlands, and Luxembourg). The results show that the SEIRD model is able to accurately predict several peaks for all the three countries, and increase and decrease in the number of infected people, with only higher RMSE for mild cases. On the other hand, the second proposed method, LSTM networks show that they are capable to predict later peaks based on the position of previous peaks with the low values of RMSE. Higher values of RMSE are observed in the forecasting of daily infected cases due the thousands of infected people per day in those countries.

\section{REFERENCES}

1. Organization World Health. Novel Coronavirus (2019-nCoV). Situation Report-6. Geneva: World Health Organization (2020). Retrieved from: https:// www.who.int/docs/default-source/coronaviruse/situation-reports/20200126sitrep-6-2019-ncov.pdf?sfvrsn=beaeee0c_4.

2. Coronavirus disease 2019. (COVID-19) Pandemic: Increased Transmission in the EU/EEA and the UK-Seventh Update. (2020). (European Centre for Disease Prevention and Control, Stockholm). Available online at: https:// www.ecdc.europa.eu/sites/default/files/documents/RRA-seventh-updateOutbreak-of-coronavirus-disease-COVID-19.pdf

3. Hethcote HW. The mathematics of infectious diseases. SIAM Rev. (2000) 42:599-653. doi: 10.1137/S0036144500371907
The match between simulated and real values can be affected by several things, such as underreporting of the number of cases, estimating initial conditions, and setting parameters. In general, if we take into account all the three countries, official and simulated values show a good match, which means that the model is showing promising results and can be further upgraded to take into account different underlying complex phenomena. Future research will include more phenomena, especially medical intervention and asymptomatic infection, mobility of people, population density, economic and social aspects, variants of concern, etc., in order to better describe the spread and development of COVID-19. We will also test the model on a greater number of countries.

\section{DATA AVAILABILITY STATEMENT}

The original contributions presented in the study are included in the article/supplementary material, further inquiries can be directed to the corresponding authors.

\section{AUTHOR CONTRIBUTIONS}

TŠ, $\mathrm{AB}$, and NF implemented the algorithms for epidemiological modeling. DC and AC collected the official data from websites. IL, SŠ, and ZC provided critical feedback and helped shape the research, analysis, and manuscript. DM and DB provided valuable discussion in the analysis of the obtained results and upgrading of the model. Article concept was created by NF and $\mathrm{ZC}$, drafted by $\mathrm{TS}$ and $\mathrm{AB}$, and written by all the authors. All the authors discussed the results and contributed to the final manuscript.

\section{FUNDING}

This research was funded by Serbian Ministry of Education, Science and Technological Development [45103-68/2020-14/200107 (Faculty of Engineering, University of Kragujevac) and 451-03-68/2020-14/200378 (University of Kragujevac, Institute for Information Technologies, Kragujevac)]. This research was also supported by the CEI project Use of Regressive Artificial Intelligence (AI) and Machine Learning (ML) Methods in Modelling of COVID-19 spread-COVIDAi.

4. Hethcote H. Three basic epidemiological models. Appl Math Ecol. (1989) 18:119-44. doi: 10.1007/978-3-642-61317-3_5

5. Hethcote HW, Van Ark JW. Modeling HIV transmission and AIDS in the United States. In: Lecture Notes in Biomath. Berlin; Heidelberg: Springer-Verlag (1992). p. 95. doi: 10.1007/978-3-642-51 477-7

6. Giordano G, Blanchini F, Bruno R, Colaneri P, Di Filippo A, Di Matteo A, et al. Modelling the COVID-19 epidemic and implementation of population-wide interventions in Italy. Nat Med. (2020) 26:85560. doi: 10.1038/s41591-020-0883-7

7. Piccolomini EL, Zama F. Monitoring Italian COVID-19 spread by an adaptive SEIRD model. medRxiv. (2020) 1-15. doi: 10.1101/2020.04.03.200 49734 
8. Zeng T, Zhang Y, Li Z, Liu X, Qiu B. Predictions of 2019-nCoV transmission ending via comprehensive methods. arXiv preprint arXiv:2002.04945 (2020). Available online at: https://arxiv.org/abs/2002.04945

9. Huang C, Wang Y, Li X, Ren L, Zhao J, Hu Y, et al. Clinical features of patients infected with 2019 novel coronavirus in Wuhan, China. Lancet. (2020) 395:497-506. doi: 10.1016/S0140-6736(20)30183-5

10. Read JM, Bridgen JRE, Cummings DAT, Ho A, Jewell CP. Novel coronavirus 2019-nCoV (COVID-19): early estimation of epidemiological parameters and epidemic size estimates. Phil Trans R Soc B. 376:20200265. doi: $10.1098 /$ rstb.2020.0265

11. Peng L, Yang W, Zhang D, Zhuge C, Hong L. Epidemic analysis of COVID-19 in China by dynamical modeling. arXiv preprint. (2020) 111 doi: 10.1101/2020.02.16.20023465

12. Tang B, Wang X, Li Q, Bragazzi NL, Tang S, Xiao Y, et al. Estimation of the transmission risk of the 2019-nCoV and its implication for public health interventions. J Clin Med. (2020) 9:462. doi: 10.3390/jcm9020462

13. Shen M, Peng Z, Guo Y, Xiao Y, Zhang L. Lockdown may partially halt the spread of 2019 novel coronavirus in Hubei province, China. Int J Infect Dis. (2020) 96:503-5. doi: 10.1016/j.ijid.2020.05.019

14. Clifford S, Pearson CA, Klepac P, Van Zandvoort K, Quilty BJ, CMMID COVID-19 working group, et al. Effectiveness of interventions targeting air travellers for delaying local outbreaks of SARS-CoV-2. J Travel Med. (2020) 27:taaa068. doi: 10.1093/jtm/taaa068

15. Xiong $\mathrm{H}$, Yan $\mathrm{H}$. Simulating the infected population and spread trend of 2019-nCoV under different policy by EIR model. Lancet. (2020) doi: 10.1101/2020.02.10.20021519

16. Tang B, Bragazzi NL, Li Q, Tang S, Xiao Y, Wu J. An updated estimation of the risk of transmission of the novel coronavirus (2019-nCov). Infect Dis Modelling. (2020) 5:248-55. doi: 10.1016/j.idm.2020.02.001

17. Chen YC, Lu PE, Chang CS, Liu TH. A time-dependent SIR model for COVID-19 with undetectable infected persons. IEEE Trans Netw Sci Eng. (2020) 7:3279-94. doi: 10.1109/TNSE.2020.3024723

18. Lin Q, Zhao S, Gao D, Lou Y, Yang S, Musa S, et al. A conceptual model for the coronavirus disease 2019 (COVID-19) outbreak in Wuhan, China with individual reaction and governmental action. Int J Inf Dis. (2020) 93:2116. doi: 10.1016/j.ijid.2020.02.058

19. Anastassopoulou C, Russo L, Tsakris A, Siettos C. Data-based analysis, modelling and forecasting of the COVID-19 outbreak. PLoS ONE. (2020) 15:e0230405. doi: 10.1371/journal.pone.0230405

20. Casella F. Can the COVID-19 epidemic be managed on the basis of daily data? IEEE Control Syst Lett. (2020) 5:1079-84. doi: 10.1109/LCSYS.2020.3009912

21. Wu JT, Leung K, Bushman M, Kishore N, Niehus R, de Salazar PM, et al. Estimating clinical severity of COVID-19 from the transmission dynamics in Wuhan, China. Nat Med. (2020) 26:506-10. doi: 10.1038/s41591-020-0822-7

22. Kucharski AJ, Russell TW, Diamond C, Liu Y, Edmunds J, Funk $S$, et al. Early dynamics of transmission and control of COVID19: a mathematical modelling study. Lancet Infect Dis. (2020) 20:e79. doi: 10.1101/2020.01.31.20019901

23. Hellewell J, Abbott S, Gimma A, Bosse N, Jarvis C, Russell T, et al. Feasibility of controlling COVID-19 outbreaks by isolation of cases and contacts. Lancet Glob Heal. (2020) 8:e488-96. doi: 10.1016/S2214-109X(20)30074-7

24. Gumel AB, Ruan S, Day T, Watmough J, Brauer F, van den Driessche P, et al. Modelling strategies for controlling SARS outbreaks. Proc R Soc B Biol Sci. (2004) 271: 2223-32. doi: 10.1098/rspb.2004.2800

25. Zahangir AM, Arifin SN, Al-Amin HM, Alam MS, Rahman MS. A spatial agent-based model of anopheles vagus for malaria epidemiology: examining the impact of vector control interventions. Malar J. (2017) 16:432. doi: 10.1186/s12936-017-2075-6

26. Perez L, Dragicevic S. An agent-based approach for modeling dynamics of contagious disease spread. Int $J$ Health Geogr. (2009) 8:50. doi: $10.1186 / 1476-072 \mathrm{X}-8-50$

27. Cuevas E. An agent-based model to evaluate the COVID19 transmission risks in facilities. Comput Biol Med. (2020) 121:103827. doi: 10.1016/j.compbiomed.2020.103827

28. Rockett RJ, Arnott A, Lam C, Sadsad R, Timms V, Gray KA, et al. Revealing COVID-19 transmission in Australia by SARS-CoV-2 genome sequencing and agent-based modeling. Nat Med. (2020) 26:1398-404. doi: 10.1038/s41591-020-1000-7
29. Inoue H, Todo Y. The propagation of economic impacts through supply chains: the case of a mega-city lockdown to prevent the spread of COVID-19. PLoS ONE. (2020) 15:e239251. doi: 10.1371/journal.pone.0239251

30. Hinch R, Probert WJ, Nurtay A, Kendall $M$, Wymant C, Hall M, et al. OpenABM-Covid19-An agent-based model for non-pharmaceutical interventions against COVID-19 including contact tracing. PLoS Comput Biol. (2021) 17:e1009146. doi: 10.1371/journal.pcbi.1009146

31. Aleta A, Martin-Corral D, Piontti AP, Ajelli M, Litvinova M, Chinazzi $\mathrm{M}$, et al. Modelling the impact of testing, contact tracing and household quarantine on second waves of COVID-19. Nat Hum Behav. (2020) 4:96471. doi: 10.1038/s41562-020-0931-9

32. Kerr CC, Mistry D, Stuart RM, Rosenfeld K, Hart GR, Núñez RC, et al. Controlling COVID-19 via test-trace-quarantine. Nat Commun. (2021) 12:2993. doi: 10.1038/s41467-021-23276-9

33. Abueg M, Hinch R, Wu N, Liu L, Probert WJ, Wu A, et al. Modeling the effect of exposure notification and non-pharmaceutical interventions on COVID-19 transmission in Washington state. npj Digit Med. (2021) 4:49. doi: 10.1038/s41746-021-00422-7

34. Silva PC, Batista PV, Lima HS, Alves MA, Guimarães FG, Silva RC. COVID-ABS: an agent-based model of COVID-19 epidemic to simulate health and economic effects of social distancing interventions. Chaos Solitons Fractals. (2020) 139:110088. doi: 10.1016/j.chaos.2020.1 10088

35. Kerr CC, Stuart RM, Mistry D, Abeysuriya RG, Rosenfeld K, Hart GR, et al. Covasim: an agent-based model of COVID-19 dynamics and interventions. PLoS Comput Biol. (2021) 17:e1009149. doi: 10.1371/journal.pcbi.10 09149

36. Schmidhuber J. Deep learning in neural networks: an overview. Neural Netw. (2015) 61:85-117. doi: 10.1016/j.neunet.2014.09.003

37. Chandra R, Shaurya G, Rishabh G. Evaluation of deep learning models for multi-step ahead time series prediction. IEEE Access. (2021) 9:8310523. doi: 10.1109/ACCESS.2021.3085085

38. Yan B, Tang X, Liu B, Wang J, Zhou Y, Zheng G, et al. An improved method for the fitting and prediction of the number of covid-19 confirmed cases based on LSTM. Comput Mater Continua. (2020) 64:147390. doi: $10.32604 / \mathrm{cmc} .2020 .011317$

39. Chimmula VK, Zhang L. Time series forecasting of COVID-19 transmission in Canada using LSTM network. Chaos Solitons Fractals. (2020) 135:109864. doi: 10.1016/j.chaos.2020.109864

40. Pereira IG, Guerin JM, Silva Júnior AG, Garcia GS, Piscitelli P, Miani A. Forecasting Covid-19 dynamics in Brazil: a data driven approach. Int J Environ Res Public Health. (2020) 17:5115. doi: 10.3390/ijerph171 45115

41. Wang P, Zheng X, Ai G, Liu D, Zhu B. Time series prediction for the epidemic trends of COVID-19 using the improved LSTM deep learning method: case studies in Russia, Peru and Iran. Chaos Solitons Fractals. (2020) 140:110214. doi: 10.1016/j.chaos.2020.110214

42. Shahid F, Zameer A, Muneeb M. Predictions for COVID-19 with deep learning models of LSTM. GRU and Bi-LST. Solitons Fractals. (2020) 140:110212. doi: $10.1016 /$ j.chaos.2020.110212

43. Yang Z, Zeng Z, Wang $\mathrm{K}$, Wong SS, Liang $\mathrm{W}$, Zanin $\mathrm{M}$, et al. Modified seir and ai prediction of the epidemics trend of COVID-19 in china under public health interventions. J Thoracic Disease. (2020) 12:165. doi: $10.21037 /$ jtd.2020.02.64

44. Kirba,ş I, Sözen A, Tuncer AD, Kazancioglu FS. Comparative analysis and forecasting of COVID-19 cases in various European countries with ARIMA. NARNN and LSTM approaches. Chaos Solitons Fractals. (2020) 138:110015. doi: 10.1016/j.chaos.2020.110015

45. Jin $\mathrm{Y}$, Yang $\mathrm{H}, \mathrm{Ji} \mathrm{W}, \mathrm{Wu} \mathrm{W}$, Chen $\mathrm{S}$, Zhang $\mathrm{W}$, et al. Virology, epidemiology, pathogenesis, and control of COVID-19. Viruses. (2020) 12:372. doi: $10.3390 / \mathrm{v} 12040372$

46. Wu Z, McGoogan JM. Characteristics of and important lessons from the coronavirus disease 2019 (COVID-19) outbreak in China. JAMA. (2020) 323:1239-42. doi: 10.1001/jama.2020.2648

47. Bastos SB, Morato MM, Cajueiro DO, Normey-Rico JE. The COVID19 (SARS-CoV-2) uncertainty tripod in Brazil: assessments on modelbased predictions with large under-reporting. Alex Eng J. (2021) 60:436380. doi: $10.1016 /$ j.aej.2021.03.004 
48. Morato MM, Pataro IM, Costa MV, Normey-Rico JE. A parametrized nonlinear predictive control strategy for relaxing COVID-19 social distancing measures in Brazil. ISA Trans. (2020) 97:269-81. doi: 10.1016/j.isatra.2020.12.012

49. Verfassungsblog (2021). Retrieved from: https://verfassungsblog.de/ belgiums-accordion-response-to-covid-19/ (accessed September 13, 2021).

50. Milovanovic DR, Jankovic SM, Ruzic Zecevic D, Folic M, Rosic N, Jovanovic D, et al. Lečenje koronavirusne bolesti (COVID-19). Med Cas. (2020) 54:2343. doi: $10.5937 / \mathrm{mckg} 54-29760$

51. Li Q, Guan X, Wu P, Wang X, Zhou L, Tong Y, et al. Early transmission dynamics in Wuhan, China, of Novel Coronavirus-Infected Pneumonia. $N$ Engl J Med. (2020) 382:1199-207. doi: 10.1056/NEJMoa2001316

52. Linton NM, Kobayashi T, Yang Y, Hayashi K, Akhmetzhanov AR, Jung S. Incubation period and other epidemiological characteristics of 2019 novel coronavirus infections with right truncation: a statistical analysis of publicly available case data. J Clin Med. (2020) 9:538. doi: 10.3390/jcm90 20538

53. Bi Q, Wu Y, Mei S, Ye C, Zou X, Zhang Z, et al. Epidemiology and transmission of COVID-19 in Shenzhen China: analysis of 391 cases and 1,286 of their close contacts. Lancet Infect Dis. (2020) 20:911-9. doi: 10.1101/2020.03.03.20028423

54. Sanche S, Lin $\mathrm{Y}, \mathrm{Xu}$ C, Romero-Severson E, Hengartner N, Ke R. The novel coronavirus, $2019-\mathrm{nCoV}$, is highly contagious and more infectious than initially estimated. Emerg Infect Dis.(2020) 26:14707. doi: $10.1101 / 2020.02 .07 .20021154$

55. Lauer SA, Grantz KH. Qifang Bi, Forrest K Jones, Zheng Q, et al. The incubation period of coronavirus disease 2019 (covid-19) from publicly reported confirmed cases: estimation and application. Annals Internal Med. (2020) 172:577-82. doi: 10.7326/M20-0504

56. Prevention Disease Control (2020). Healthcare Professionals: Frequently Asked Questions and Answers. Atlanta: Centers for Disease Control and Prevention. Retrieved from: https://www.cdc.gov/coronavirus/2019-ncov/hcp/faq.html.

57. Woelfel R, Corman VM, Guggemos W, Seilmaier M, Zange S, Mueller MA, et al. Clinical presentation and virological assessment of hospitalized cases of coronavirus disease 2019 in a travel-associated transmission cluster. medRxiv. (2020). doi: 10.1101/2020.03.05.20030502

58. Guan WJ, Ni ZY, Hu Y, Liang WH, Ou CQ, He JX, et al. Clinical characteristics of coronavirus disease 2019 in China. N Engl J Med. (2020) 382:170820. doi: 10.1056/NEJMoa2002032

59. Liu Y, Funk S, Flasche S. The Contribution of Pre-symptomatic Transmission to the COVID-19 Outbreak. (2020). Available online at: https://cmmid.github.io/ topics/covid19/pre-symptomatic-transmission.html

60. Russell TW, Hellewell J, Jarvis CI, Zandvoort K, Abbott S, Ratnayake R, et al. Estimating the Infection and Case Fatality Ratio for COVID-19 Using AgeAdjusted Data From the Outbreak on the Diamond Princess Cruise Ship. (2020). Available online at: https://cmmid.github.io/topics/covid19/diamond_cruise_ cfr_estimates.html

61. Hauser A, Counotte MJ, Margossian CC, Konstantinoudis G, Low N, Althaus CL, et al. Estimation of SARS-CoV-2 mortality during the early stages of an epidemic: a modeling study in Hubei, China, and six regions in Europe. PLoS Med. (2020) 17:e1003189. doi: 10.1371/journal.pmed.10 03189

62. Cao B, Wang Y, Wen D, Liu W, Wang J, Fan G, et al. A trial of lopinavir-ritonavir in adults hospitalized with severe Covid19. N Engl J Med. (2020) 382:1787-99. doi: 10.1056/NEJMoa2 001282

63. Callaway E. Coronavirus vaccines: five key questions as trials begin. Nature. (2020) 579:481. doi: 10.1038/d41586-020-00798-8

64. Tindale L, Coombe M, Stockdale JE, Garlock E, Lau WYV, Saraswat M, et al. Transmission interval estimates suggest pre-symptomatic spread of COVID-19. eLife. (2020) 9:e57149. doi: 10.7554/eLife.57149

65. Huang C, Wang Y, Li X, Ren L, Zhao J, Hu Y, et al. Clinical features of patients infected with 2019 novel coronavirus in Wuhan, China. Lancet Respir Med. (2020) 8:475-81.

66. Zhou F, Yu T, Du R, Fan G, Liu Y, Liu Z, et al. Clinical course and risk factors for mortality of adult inpatients with COVID-19 in Wuhan, China: a retrospective cohort study. Lancet. (2020) 395:105462. doi: 10.1016/S0140-6736(20)30566-3
67. Ritchie H, Mathieu E, Rodés-Guirao L, Appel C, Giattino C, Ortiz-Ospina E, et al. Coronavirus Pandemic (COVID-19). (2020). Retrieved from: https:// ourworldindata.org/coronavirus (accessed October 12, 2021).

68. Infectious Diseases Data Explorations and Visualizations. (2020). Retrieved from: https://epistat.wiv-isp.be/covid/ (accessed October 12, 2021).

69. The luxembourgish data platform. (2020). Retrieved from: https://data.public. $\mathrm{lu} / \mathrm{fr} /$ (accessed October 12, 2021).

70. Shim E. Delay-adjusted age-specific COVID-19 case fatality rates in a high testing setting: South Korea, February 2020 to February 2021. Int J Environ Res Public Health. (2021) 18:5053. doi: 10.3390/ijerph18105053

71. Kobayashi T, Jung SM, Linton NM, Kinoshita R, Hayashi K, Miyama T, et al. Communicating the risk of death from novel coronavirus disease (COVID19). J Clin Med. (2020) 9:580. doi: $10.3390 / \mathrm{jcm} 9020580$

72. Faes C, Abrams S, Van Beckhoven D, Meyfroidt G, Vlieghe E, Hens N. Time between symptom onset, hospitalisation and recovery or death: statistical analysis of Belgian COVID-19 patients. Int J Environ Res Public Health. (2020) 17:7560. doi: 10.3390/ijerph17207560

73. Hochreiter S, Schmidhuber J. Long short-term memory. Neural Comput. (1997) 9:1735-80. doi: 10.1162/neco.1997.9.8.1735

74. Chandra R, Jain A, Chauhan DS. Deep Learning via LSTM Models for COVID19 Infection Forecasting in India. arXiv:2101.11881 (2021).

75. Köhler J, Schwenkel L, Koch A, Berberich J, Pauli P, Allgöwer F. Robust and optimal predictive control of the COVID-19 outbreak. Ann Rev Control. (2021) 51:525-39. doi: 10.1016/j.arcontrol.2020. 11.002

76. Kantner M, Koprucki T. Beyond just "flattening the curve": Optimal control of epidemics with purely non-pharmaceutical interventions. J Math Ind. (2020) 10:23. doi: 10.1186/s13362-020-00091-3

77. Alleman T, Torfs E, Nopens I. COVID-19: From Model Prediction to Model Predictive Control. Retrieved September (2020). p. 12 (2021). Available online at: https://biomath.ugent.be/sites/default/files/2020-04/Alleman_etal_v2.pdf.

78. Imai N, Dorigatti I, Cori A, Donnelly C, Riley S, Ferguson N. Estimating the Potential Total Number of Novel Coronavirus cases in Wuhan City, China. Medical Research Council (MRC) (2020).

79. Poletto C, Scarpino SV, Volz EM. Applications of predictive modelling early in the COVID-19 epidemic. Lancet Digit Health. (2020) 2:4989. doi: 10.1016/S2589-7500(20)30196-5

80. Korolev I. Identification and estimation of the SEIRD epidemic model for COVID-19. J Econom. (2020) 220:63-85. doi: 10.1016/j.jeconom.2020.07.038

81. Vadyala SR, Betgeri SN, Sherer EA, Amritphale A. Prediction of the number of covid-19 confirmed cases based on K-meansLSTM. Array. (2021) 11:100085. doi: 10.1016/j.array.2021.1 00085

82. Ayyoubzadeh SM, Ayyoubzadeh SM, Zahedi H, Ahmadi M, Kalhori SR. Predicting COVID-19 incidence through analysis of google trends data in iran: data mining and deep learning pilot study. JMIR Public Health Surveill. (2020) 6:e18828. doi: 10.2196/18828

83. Griffin S. Covid-19: asymptomatic cases may not be infectious, Wuhan study indicates. $\mathrm{Br}$ Med J Publishing Group. (2020) 371:m4695. doi: 10.1136/bmj.m4695

84. Lekone PE, Finkenstädt BF. Statistical inference in a stochastic epidemic SEIR model with control intervention: ebola as a case study. Biometrics. (2006) 62:1170-7. doi: 10.1111/j.1541-0420.2006.00609.x

85. Melin P, Castillo O. Spatial and temporal spread of the COVID-19 pandemic using self organizing neural networks and a fuzzy fractal approach. Sustainability. (2021) 13:8295. doi: 10.3390/su13158295

86. Khan SD, Alarabi L, Basalamah S. Toward smart lockdown: a novel approach for COVID-19 hotspots prediction using a deep hybrid neural network. Computers. (2020) 9:99. doi: 10.3390/computers9040099

87. International, TomTom. The Effect of the COVID-19 Confinement on Belgian Mobility. (2020). Retrieved from: https://www.tomtom.com/covid19/country/belgium/ (accessed September 13, 2021).

88. Fonseca Casas P, Garcia Carrasco V, Garcia Subirana J. SEIRD COVID-19 formal characterization and model comparison validation. Appl Sci. (2020) 10:5162. doi: 10.3390/app10155162

89. Google See How Your Community is Moving Around Differently Due to COVID-19 (2020). Available online at: https://www.google.com/covid19/ mobility/ (accessed April 20, 2020). 
90. Genomic surveillance of SARS-Co, V.-2 in Belgium (2021). Available online at: https://assets.uzleuven.be/files/2021-08/genomic_surveillance_update_ 210824.pdf.

91. National Institute for Public Health and the Environment (2021). Retrieved from: https://www.rivm.nl/en/coronavirus-covid-19/virus-sars-cov-2/ variants (accessed September 13, 2021).

92. Laboratoire national de santé (2021). Retrieved from: https://nns.lu/en/ departement/department-of-microbiology/revilux/ (accessed September 13, 2021).

93. Benjamin DJ, Cesarini D, Chabris CF, Glaeser EL, Laibson DI, Guðnason V, et al. The promises and pitfalls of genoeconomics. Annu Rev Econ. (2012) 4:627-62. doi: 10.1146/annurev-economics-080511-110939

94. Nakanishi T, Pigazzini S, Degenhardt F, Cordioli M, Butler-Laporte G, Maya-Miles D, et al. Age-dependent impact of the major common genetic risk factor for COVID-19 on severity and mortality. medRxiv [Preprint]. (2021) 2021.03.07.21252875. doi: 10.1101/2021.03.07.21 252875
Conflict of Interest: The authors declare that the research was conducted in the absence of any commercial or financial relationships that could be construed as a potential conflict of interest.

Publisher's Note: All claims expressed in this article are solely those of the authors and do not necessarily represent those of their affiliated organizations, or those of the publisher, the editors and the reviewers. Any product that may be evaluated in this article, or claim that may be made by its manufacturer, is not guaranteed or endorsed by the publisher.

Copyright (c) 2021 Šušteršič, Blagojević, Cvetković, Cvetković, Lorencin, Šegota, Milovanović, Baskić, Car and Filipović. This is an open-access article distributed under the terms of the Creative Commons Attribution License (CC BY). The use, distribution or reproduction in other forums is permitted, provided the original author(s) and the copyright owner(s) are credited and that the original publication in this journal is cited, in accordance with accepted academic practice. No use, distribution or reproduction is permitted which does not comply with these terms. 